Supporting Information for

\title{
Which is More Likely in Trichodiene Biosynthesis: Hydride or Proton Transfer?
}

\author{
Young J. Hong and Dean J. Tantillo* \\ Department of Chemistry \\ University of California, Davis \\ One Shields Avenue \\ Davis, CA 95616 \\ tantillo@chem.ucdavis.edu
}

Coordinates and energies for all computed structures, along with the full Gaussian citation. 
Full citation for Gaussian (ref 4):

Gaussian 03, Revision B.04,

M. J. Frisch, G. W. Trucks, H. B. Schlegel, G. E. Scuseria,

M. A. Robb, J. R. Cheeseman, J. A. Montgomery, Jr., T. Vreven,

K. N. Kudin, J. C. Burant, J. M. Millam, S. S. lyengar, J. Tomasi,

V. Barone, B. Mennucci, M. Cossi, G. Scalmani, N. Rega,

G. A. Petersson, H. Nakatsuji, M. Hada, M. Ehara, K. Toyota,

R. Fukuda, J. Hasegawa, M. Ishida, T. Nakajima, Y. Honda, O. Kitao,

H. Nakai, M. Klene, X. Li, J. E. Knox, H. P. Hratchian, J. B. Cross,

C. Adamo, J. Jaramillo, R. Gomperts, R. E. Stratmann, O. Yazyev,

A. J. Austin, R. Cammi, C. Pomelli, J. W. Ochterski, P. Y. Ayala,

K. Morokuma, G. A. Voth, P. Salvador, J. J. Dannenberg,

V. G. Zakrzewski, S. Dapprich, A. D. Daniels, M. C. Strain,

O. Farkas, D. K. Malick, A. D. Rabuck, K. Raghavachari,

J. B. Foresman, J. V. Ortiz, Q. Cui, A. G. Baboul, S. Clifford,

J. Cioslowski, B. B. Stefanov, G. Liu, A. Liashenko, P. Piskorz,

I. Komaromi, R. L. Martin, D. J. Fox, T. Keith, M. A. Al-Laham,

C. Y. Peng, A. Nanayakkara, M. Challacombe, P. M. W. Gill,

B. Johnson, W. Chen, M. W. Wong, C. Gonzalez, and J. A. Pople,

Gaussian, Inc., Pittsburgh PA, 2003. 
1] Bisabolyl-I

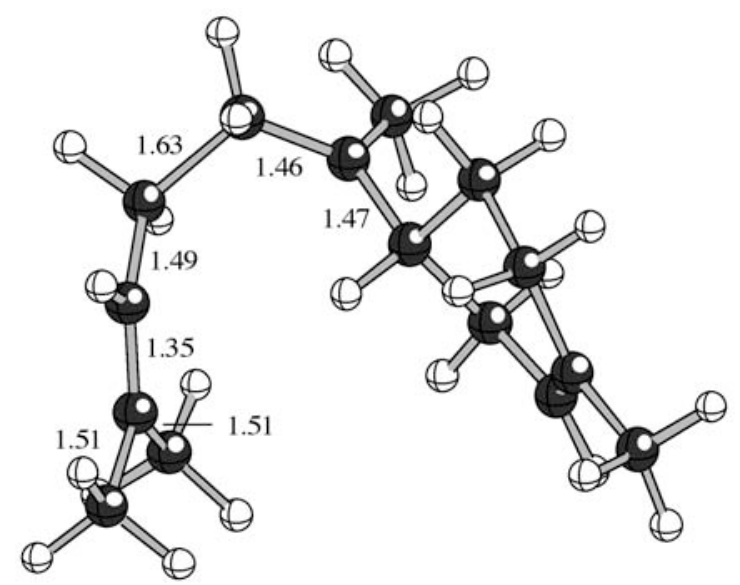

B3LYP/6-31+G[d,p]: -586.4146838 hartrees [-367975.2140845 kcal/mol] Imaginary frequencies: none

Zero-point correction $=0.363052$ [Hartree/Particle)

mPW1PW91/6-31+G[d,p]//B3LYP/6-31+G[d,p]: -586.2752278 hartrees [-367887.7054445 kcal/mol]

Coordinates:

\begin{tabular}{|c|c|c|c|c|}
\hline \multirow{2}{*}{$\begin{array}{l}\text { Center } \\
\text { number }\end{array}$} & Atomic & \multicolumn{3}{|c|}{ Coordinates [Angstroms] } \\
\hline & number & 8 & $\mathbf{Y}$ & Z \\
\hline 1 & 6 & 2.294619 & 0.628322 & 1.173158 \\
\hline 2 & 6 & 2.970260 & 0.787724 & 0.024316 \\
\hline 3 & 6 & 2.654425 & -0.041777 & -1.201406 \\
\hline 4 & 6 & 1.731369 & -1.236013 & -0.934600 \\
\hline 5 & 6 & 0.553025 & -0.774265 & 0.026528 \\
\hline 6 & 6 & 1.162984 & -0.350617 & 1.373226 \\
\hline 7 & 1 & 2.224982 & $0.605317-$ & -1.979775 \\
\hline 8 & 1 & 2.557130 & 1.237877 & 2.034815 \\
\hline 9 & 1 & 1.342710 & -1.636130 & -1.874960 \\
\hline 10 & 1 & 2.278121 & -2.038710 & -0.427973 \\
\hline 11 & 1 & 3.588099 & -0.433500 & -1.627816 \\
\hline 12 & 6 & -1.440478 & -1.893679 & -1.027790 \\
\hline 13 & 6 & -2.691605 & -0.946632 & -0.579133 \\
\hline 14 & 1 & -1.834117 & -2.901840 & -1.170038 \\
\hline 15 & 1 & -1.073306 & -1.484886 & 6 -1.971858 \\
\hline 16 & 6 & -2.495801 & 0.494196 & -0.897898 \\
\hline 17 & 1 & -3.507760 & -1.359740 & -1.181503 \\
\hline 18 & 1 & -2.942733 & -1.134235 & $5 \quad 0.466492$ \\
\hline 19 & 6 & -2.515625 & 1.541814 & -0.041576 \\
\hline 20 & 6 & -0.453615 & -1.839979 & 0.040170 \\
\hline 21 & 6 & -2.405946 & 2.951743 & -0.562453 \\
\hline 22 & 1 & -3.296945 & 3.530870 & -0.290204 \\
\hline 23 & 1 & -1.550849 & 3.468415 & -0.108855 \\
\hline
\end{tabular}




\begin{tabular}{|c|c|c|c|}
\hline 24 & 1 & -2.296328 & $2.985445-1.648967$ \\
\hline 25 & 6 & -2.683878 & $1.431526 \quad 1.451372$ \\
\hline 26 & 1 & -3.631504 & 1.8925391 .756952 \\
\hline 27 & 1 & -2.681426 & $0.406221 \quad 1.826935$ \\
\hline 28 & 1 & -1.893014 & $1.990441 \quad 1.965940$ \\
\hline 29 & 1 & -2.387700 & $0.714523-1.959935$ \\
\hline 30 & 1 & 0.094914 & $0.094333-0.476140$ \\
\hline 31 & 6 & -0.511324 & $-2.837352 \quad 1.130001$ \\
\hline 32 & 1 & 0.430764 & $-3.409785 \quad 1.121626$ \\
\hline 33 & 1 & -0.532640 & $-2.351991 \quad 2.113636$ \\
\hline 34 & 1 & -1.343412 & $-3.535122 \quad 1.031417$ \\
\hline 35 & 1 & 1.521224 & $-1.229945 \quad 1.926503$ \\
\hline 36 & 1 & 0.385929 & $0.108236 \quad 1.998002$ \\
\hline 37 & 6 & 4.073449 & $1.800258-0.134457$ \\
\hline 38 & 1 & 5.024923 & $1.309212-0.373245$ \\
\hline 39 & 1 & 3.858248 & $2.489022-0.961283$ \\
\hline 40 & 1 & 4.213545 & $2.391820 \quad 0.773496$ \\
\hline
\end{tabular}

2) TS bisabolyl-I-to-Ca

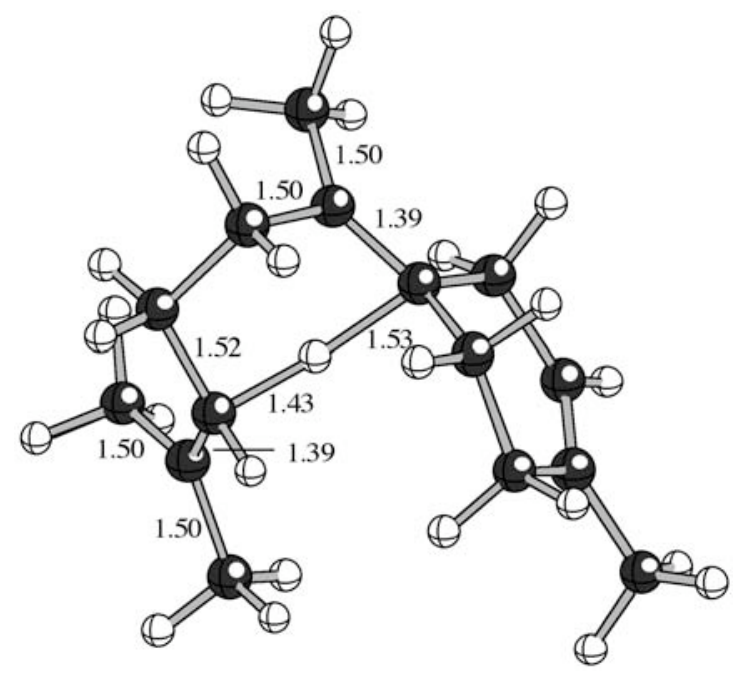

B3LYP/6-31+G[d,p]: -586.3972994 hartrees [-367964.3053735 kcal/mol]

Imaginary frequencies: $1[-923.08601 / \mathrm{cm}]$

Zero-point correction $=0.358495$ (Hartree/Particle $)$

mPW1PW91/6-31+G[d,p]//B3LYP/6-31+G[d,p]: -586.2627619 hartrees [-367879.88309225 kcal/mol]

Coordinates:

\begin{tabular}{lllll} 
Center & \multicolumn{1}{l}{ Atomic } & \multicolumn{3}{c}{ Coordinates [fingstroms] } \\
number & number & X & $Y$ & $Z$ \\
\hline 1 & 6 & 2.284004 & -0.468784 & 1.199884 \\
2 & 6 & 2.775645 & 0.058111 & 0.069191
\end{tabular}




$\begin{array}{ccccc}3 & 6 & 2.071747 & -0.139263 & -1.253902 \\ 4 & 6 & 1.015805 & -1.255174 & -1.225047 \\ 5 & 6 & 0.163730 & -1.275921 & 0.045398 \\ 6 & 6 & 1.010124 & -1.272245 & 1.313786 \\ 7 & 1 & 1.624236 & 0.813735 & -1.573978 \\ 8 & 1 & 2.826043 & -0.327644 & 2.132831 \\ 9 & 1 & 0.411959 & -1.226958 & -2.133349 \\ 10 & 1 & 1.537531 & -2.222271 & -1.231340 \\ 11 & 1 & 2.809905 & -0.379618 & -2.029032 \\ 12 & 6 & -2.056433 & -1.492060 & -1.113638 \\ 13 & 6 & -2.564899 & -0.002356 & -1.026034 \\ 14 & 1 & -2.921267 & -2.159069 & -1.082894 \\ 15 & 1 & -1.551118 & -1.644031 & -2.068500 \\ 16 & 6 & -1.407791 & 0.960734 & -0.832390 \\ 17 & 1 & -3.087529 & 0.239274 & -1.956869 \\ 18 & 1 & -3.295813 & 0.082131 & -0.218448 \\ 19 & 6 & -1.363483 & 2.014780 & 0.070547 \\ 20 & 6 & -1.159534 & -1.710467 & 0.066541 \\ 21 & 6 & -0.323464 & 3.081184 & -0.064515 \\ 22 & 1 & -0.813180 & 4.062692 & -0.115910 \\ 23 & 1 & 0.315584 & 3.108794 & 0.826956 \\ 24 & 1 & 0.299979 & 2.957245 & -0.951301 \\ 25 & 6 & -2.293756 & 2.151552 & 1.234199 \\ 26 & 1 & -3.043165 & 2.920064 & 0.995598 \\ 27 & 1 & -2.831543 & 1.235170 & 1.479495 \\ 28 & 1 & -1.761133 & 2.508186 & 2.121479 \\ 29 & 1 & -0.774584 & 1.086266 & -1.712765 \\ 30 & 1 & -0.577065 & 0.057093 & -0.106576 \\ 31 & 6 & -1.848189 & -2.188808 & 1.311495 \\ 32 & 1 & -1.957186 & -3.280083 & 1.223282 \\ 33 & 1 & -1.303911 & -1.993880 & 2.234416 \\ 34 & 1 & -2.864228 & -1.790079 & 1.397334 \\ 35 & 1 & 1.253419 & -2.314560 & 1.576120 \\ 36 & 1 & 0.430536 & -0.891769 & 2.162627 \\ 37 & 6 & 4.049242 & 0.861743 & 0.035613 \\ 38 & 1 & 4.806172 & 0.367427 & -0.586042 \\ 39 & 1 & 3.881605 & 1.853006 & -0.405291 \\ 40 & 1 & 4.468258 & 1.000727 & 1.035388 \\ -----------------------------------------\end{array}$

IRC: TS[bisabolyl-I-to-Ca) -> bisabolyl-I 

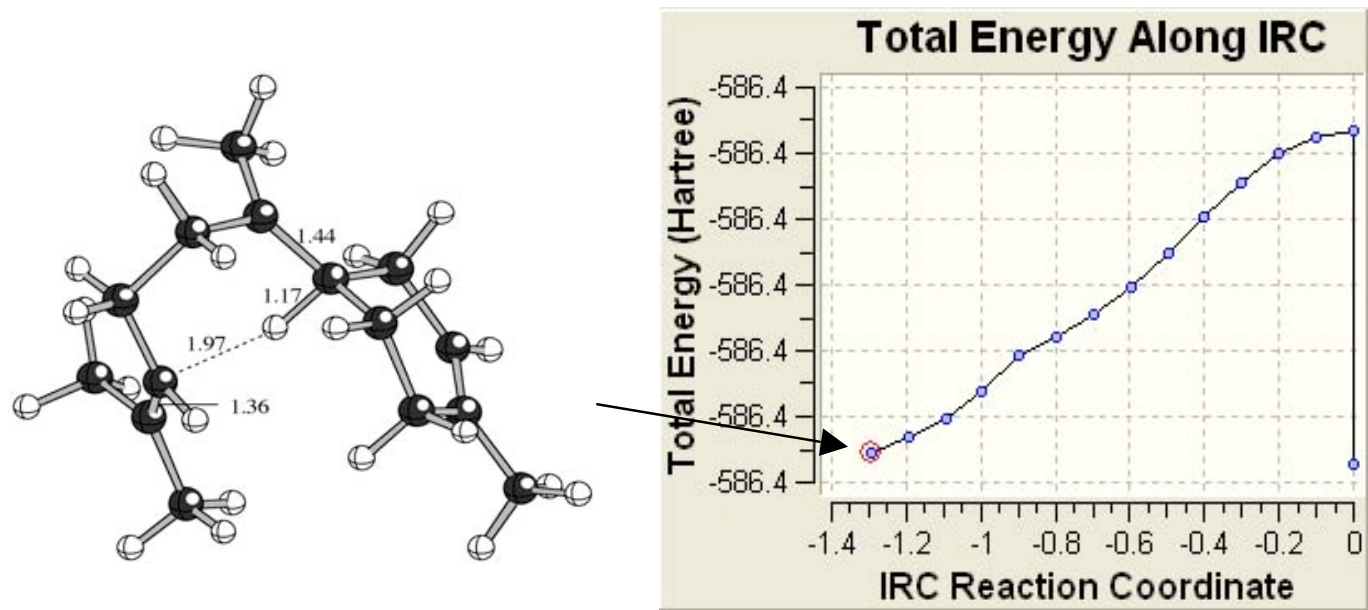

IRC: TS(bisabolyl-I-to-Ca) $\rightarrow$ Ca

Total Energy Along IRC

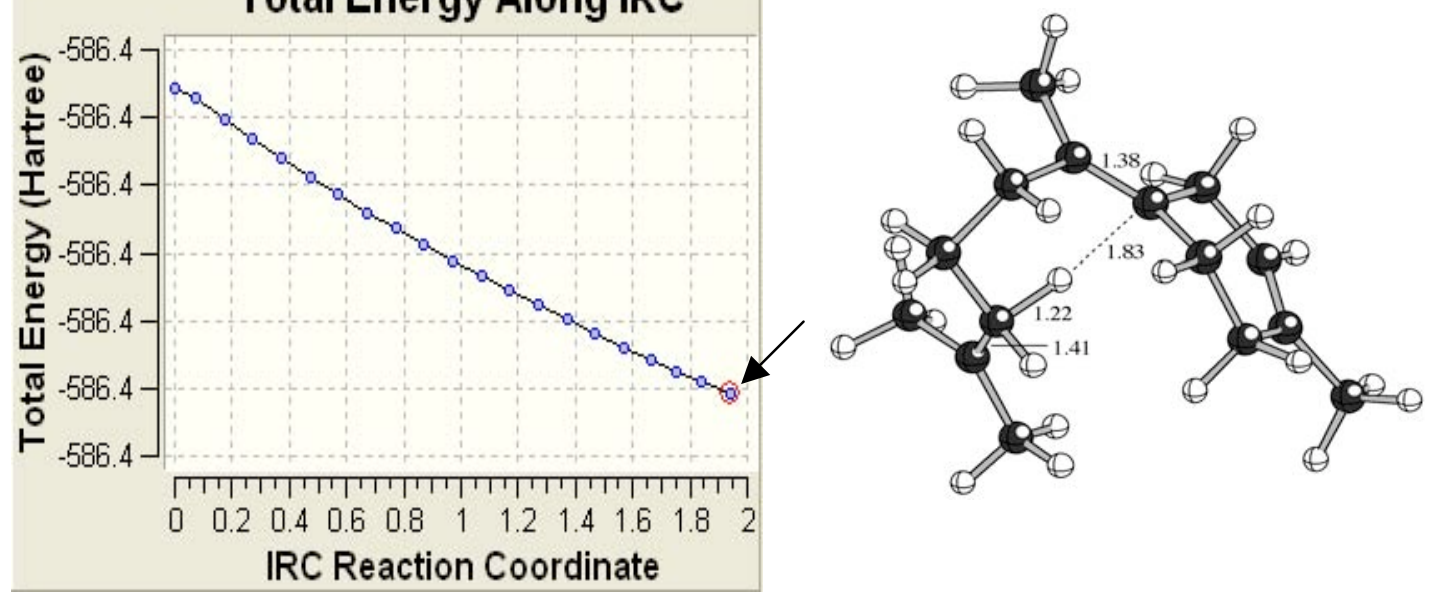

3) Ca

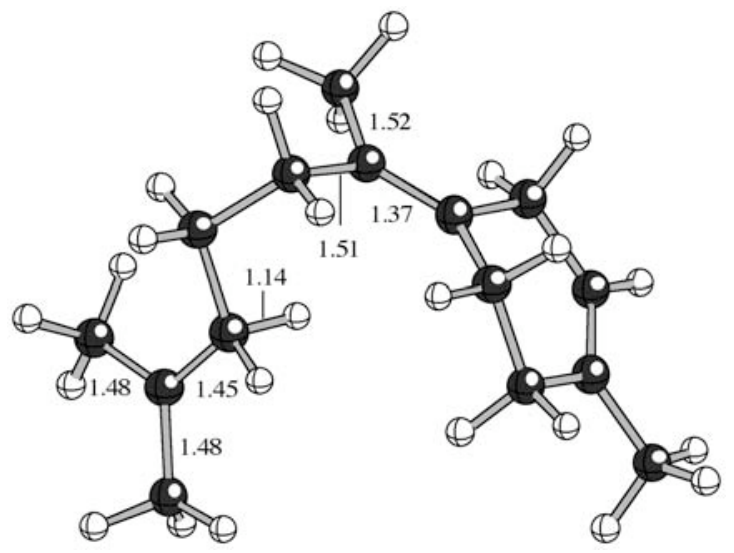

B3LYP/6-31+G[d,p]: -586.4067226 hartrees [-367970.2184315 kcal/mol]

Imaginary frequencies: none 
Zero-point correction = 0.361338 (Hartree/Particle)

mPW1PW91/6-31+G[d,p]//B3LYP/6-31+G[d,p]: -586.2685837 hartrees [-367883.53627175 kcal/mol]

Coordinates:

\begin{tabular}{|c|c|c|c|c|}
\hline \multirow{2}{*}{$\begin{array}{l}\text { Center } \\
\text { number }\end{array}$} & ftomic & \multicolumn{3}{|c|}{ Coordinates [Angstroms] } \\
\hline & number & X & $\mathbf{Y}$ & Z \\
\hline 1 & 6 & -2.936450 & -0.041459 & -1.041774 \\
\hline 2 & 6 & -2.958568 & 0.966144 & -0.157805 \\
\hline 3 & 6 & -2.075372 & 0.932395 & 1.070325 \\
\hline 4 & 6 & -1.555130 & -0.482126 & 1.393502 \\
\hline 5 & 6 & -1.025293 & -1.196944 & 0.172418 \\
\hline 6 & 6 & -2.032826 & -1.250019 & -0.956646 \\
\hline 7 & 1 & -1.234116 & 1.632177 & 0.937361 \\
\hline 8 & 1 & -3.597730 & -0.010427 & -1.905665 \\
\hline 9 & 1 & -0.832570 & -0.438894 & 2.210760 \\
\hline 10 & 1 & -2.406446 & -1.071168 & 1.766002 \\
\hline 11 & 1 & -2.633162 & 1.310770 & 1.936188 \\
\hline 12 & 6 & 1.211337 & -1.845902 & 1.189854 \\
\hline 13 & 6 & 2.430029 & -0.964231 & 0.818783 \\
\hline 14 & 1 & 1.571493 & -2.869077 & 1.351383 \\
\hline 15 & 1 & 0.800165 & -1.507475 & 2.142805 \\
\hline 16 & 6 & 1.940687 & 0.468857 & 0.531397 \\
\hline 17 & 1 & 3.162432 & -0.947726 & 1.633366 \\
\hline 18 & 1 & 2.929949 & -1.383656 & -0.059234 \\
\hline 19 & 6 & 2.764903 & 1.392518 & -0.215361 \\
\hline 20 & 6 & 0.180027 & -1.835731 & 0.083594 \\
\hline 21 & 6 & 2.653287 & 2.847491 & 0.015167 \\
\hline 22 & 1 & 3.641347 & 3.229837 & 0.320698 \\
\hline 23 & 1 & 2.439442 & 3.363669 & -0.931268 \\
\hline 24 & 1 & 1.915861 & 3.118228 & 0.770918 \\
\hline 25 & 6 & 3.720750 & 0.923328 & -1.242914 \\
\hline 26 & 1 & 4.642307 & 0.613323 & -0.720625 \\
\hline 27 & 1 & 3.363918 & 0.031500 & -1.766379 \\
\hline 28 & 1 & 3.995158 & 1.706057 & -1.952553 \\
\hline 29 & 1 & 1.514632 & 0.948095 & 1.419130 \\
\hline 30 & 1 & 1.033592 & 0.315816 & -0.142179 \\
\hline 31 & 6 & 0.557771 & -2.644944 & -1.147234 \\
\hline 32 & 1 & -0.128445 & -3.489071 & | -1.285124 \\
\hline 33 & 1 & 0.524472 & -2.061942 & -2.074390 \\
\hline 34 & 1 & 1.562804 & -3.065687 & -1.056205 \\
\hline 35 & 1 & -2.649675 & -2.156911 & -0.824426 \\
\hline 36 & 1 & -1.535948 & -1.392682 & -1.922229 \\
\hline 37 & 6 & -3.848504 & 2.171191 & -0.314863 \\
\hline 38 & 1 & -4.558996 & 2.246363 & 0.518082 \\
\hline 39 & 1 & -3.261749 & 3.099492 & -0.310295 \\
\hline 40 & 1 & -4.418621 & 2.135985 & -1.246772 \\
\hline
\end{tabular}


4) TS Ca-to-Cb

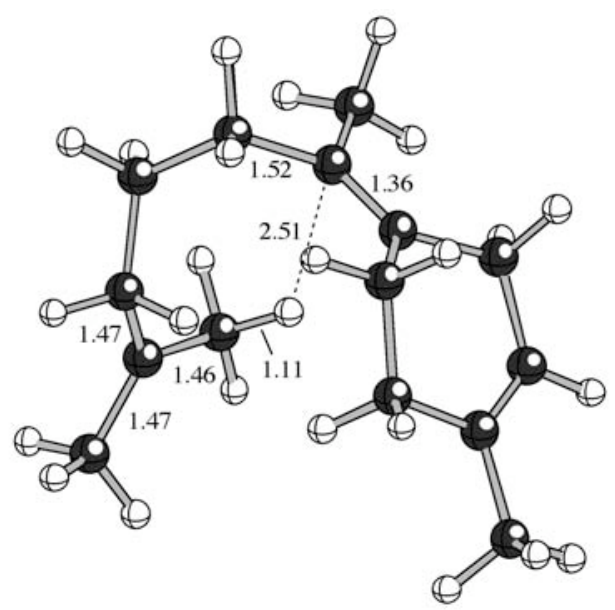

B3LYP/6-31+G[d,p]: -586.3994222 hartrees [-367965.6374305 kcal/mol]

Imaginary frequencies: $1[-54.93281 / \mathrm{cm}]$

Zero-point correction = 0.361407 (Hartree/Particle)

mPW1PW91/6-31+G[d,p]//B3LYP/6-31+G[d,p]: -586.2618917 hartrees [-367879.33704175 kcal/mol]

Coordinates:

\begin{tabular}{|c|c|c|c|c|}
\hline Center & Atomic & & ordinates [fin & igstroms] \\
\hline number & numbe & 8 & $\mathbf{Y}$ & $\mathbf{Z}$ \\
\hline 1 & 6 & 0.709598 & -1.324523 & -0.182286 \\
\hline 2 & 6 & -1.012421 & -2.508406 & 1.251862 \\
\hline 3 & 1 & -2.043366 & -2.255568 & 1.528380 \\
\hline 4 & 1 & -1.012065 & -3.588626 & 1.054509 \\
\hline 5 & 1 & -0.384103 & -2.342717 & 2.127705 \\
\hline 6 & 6 & -0.564751 & -1.761779 & 0.009981 \\
\hline 7 & 6 & -1.657274 & -1.677941 & -1.044754 \\
\hline 8 & 6 & -2.091507 & 1.635661 & 0.371233 \\
\hline 9 & 6 & -2.760785 & -0.629037 & -0.772235 \\
\hline 10 & 1 & -2.156044 & -2.654268 & -1.100257 \\
\hline 11 & 1 & -1.246671 & -1.493809 & -2.040582 \\
\hline 12 & 6 & -2.282526 & 0.849272 & -0.851916 \\
\hline 13 & 1 & -3.547136 & -0.747384 & -1.524091 \\
\hline 14 & 1 & -3.243092 & -0.817628 & 0.192584 \\
\hline 15 & 6 & -2.257890 & 3.097068 & 0.324600 \\
\hline 16 & 1 & -3.264499 & 3.289718 & 0.744616 \\
\hline 17 & 1 & -1.559891 & 3.619844 & 0.985092 \\
\hline 18 & 1 & -2.248846 & 3.508515 & -0.686744 \\
\hline 19 & 6 & -1.662308 & 0.988822 & 1.606974 \\
\hline 20 & 1 & -1.433165 & 1.677952 & 2.418755 \\
\hline 21 & 1 & -2.403731 & 0.244942 & 1.931776 \\
\hline
\end{tabular}




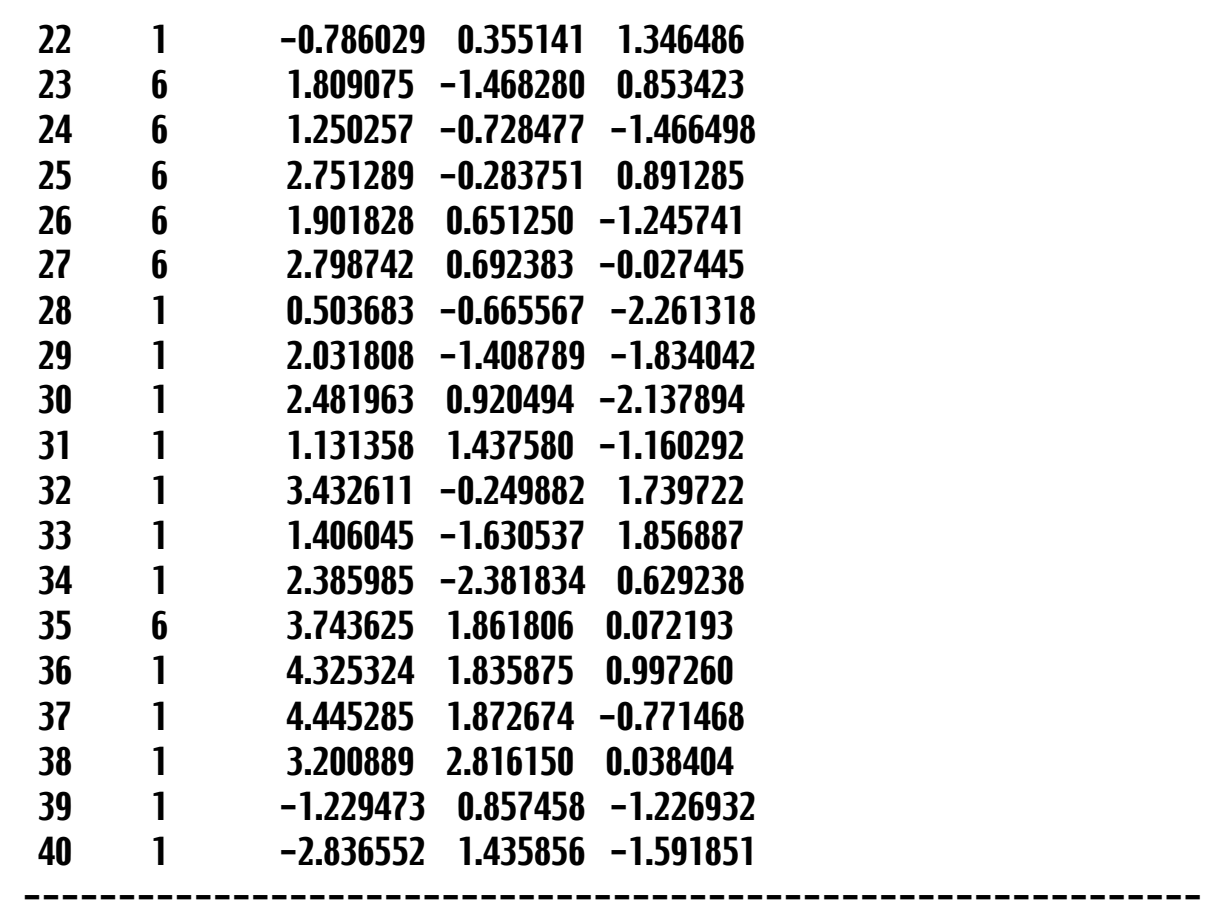

IRC: TS[Ca-to-Cb] $\rightarrow$ Ca

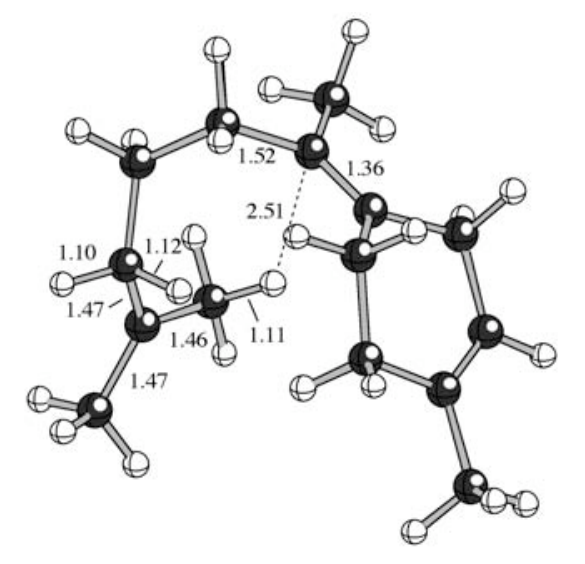

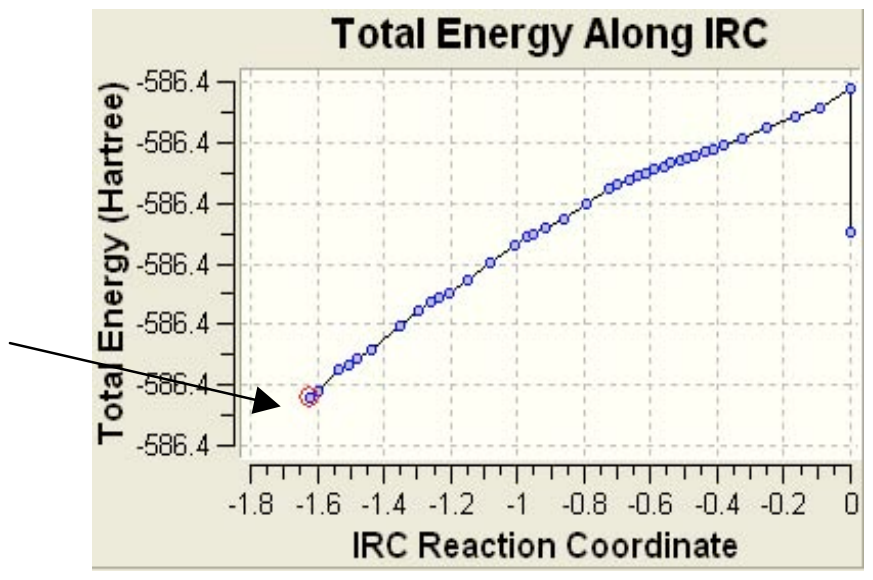

IRC: TS(Ca-to-Cb] $\rightarrow$ Cb 


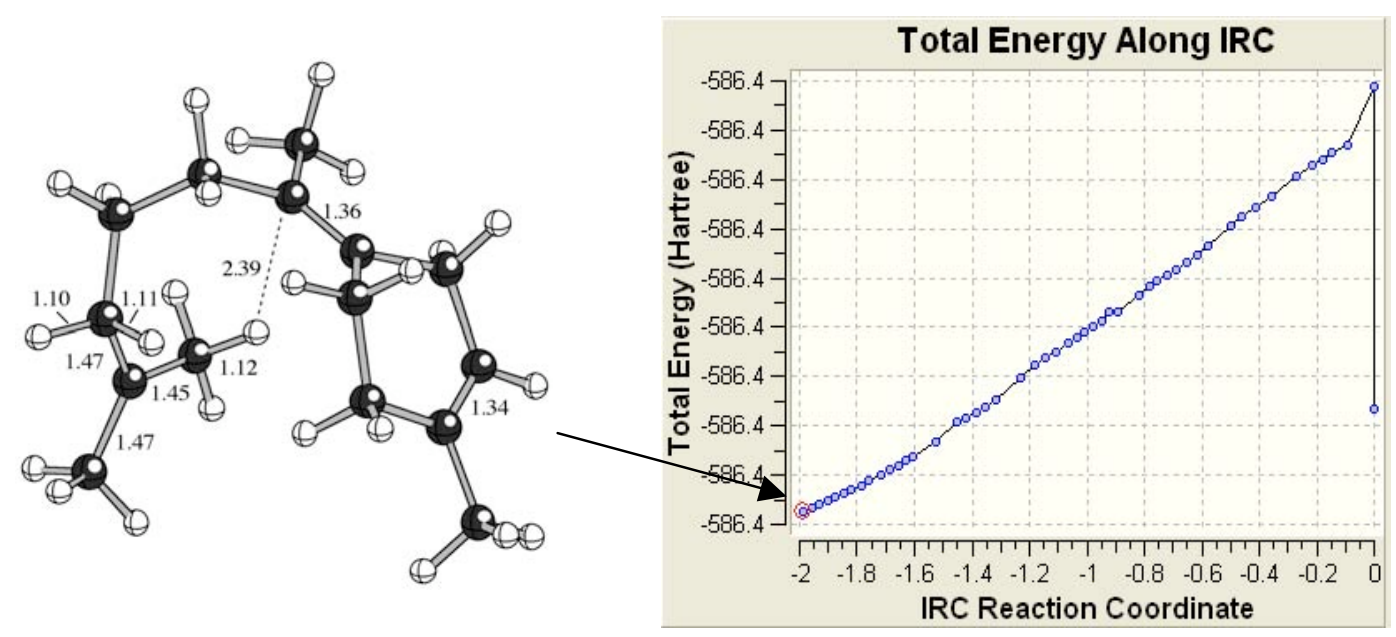

5) Cb

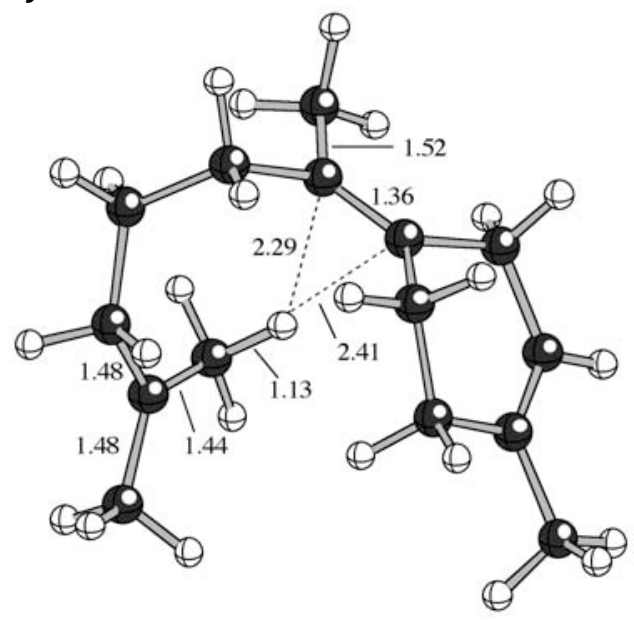

B3LYP/6-31+G[d,p]: -586.3998298 hartrees [-367965.8931995 kcal/mol]

Imaginary frequencies: none

Zero-point correction $\mathbf{0} 0.361237$ (Hartree/Particle)

mPW1PW91/6-31+G[d,p]//B3LYP/6-31+G[d,p]: -586.262534 hartrees [-367879.740085 kcal/mol]

Coordinates:

\begin{tabular}{|c|c|c|c|c|}
\hline Center & Atomic & & ordinates [fin & ngstroms] \\
\hline number & number & & $Y \quad Y$ & Z \\
\hline 1 & 6 & -0.590618 & -1.345602 & 0.293722 \\
\hline 2 & 6 & 1.086309 & -2.581597 & -1.150153 \\
\hline 3 & 1 & 2.040910 & -2.256756 & -1.582441 \\
\hline 4 & 1 & 1.241954 & -3.618568 & -0.824176 \\
\hline 5 & 1 & 0.349718 & -2.601915 & -1.953127 \\
\hline 6 & 6 & 0.691048 & -1.725048 & 0.035589 \\
\hline 7 & 6 & 1.861390 & -1.466096 & 0.979766 \\
\hline 8 & 6 & 1.852430 & 1.716207 & -0.456474 \\
\hline
\end{tabular}




$\begin{array}{ccccc}9 & 6 & 2.952700 & -0.467869 & 0.516777 \\ 10 & 1 & 2.369794 & -2.428215 & 1.126031 \\ 11 & 1 & 1.518667 & -1.161882 & 1.972254 \\ 12 & 6 & 2.596090 & 1.028756 & 0.619287 \\ 13 & 1 & 3.822157 & -0.617299 & 1.164882 \\ 14 & 1 & 3.304506 & -0.703121 & -0.494149 \\ 15 & 6 & 1.667448 & 3.177165 & -0.359345 \\ 16 & 1 & 2.320762 & 3.632382 & -1.124592 \\ 17 & 1 & 0.650550 & 3.466033 & -0.649757 \\ 18 & 1 & 1.932747 & 3.594042 & 0.613353 \\ 19 & 6 & 1.275938 & 0.990286 & -1.564104 \\ 20 & 1 & 0.774958 & 1.604588 & -2.311243 \\ 21 & 1 & 1.990407 & 0.291614 & -2.017536 \\ 22 & 1 & 0.553849 & 0.269491 & -1.080768 \\ 23 & 6 & -1.767662 & -1.690894 & -0.597660 \\ 24 & 6 & -1.044404 & -0.586602 & 1.523929 \\ 25 & 6 & -2.779943 & -0.571591 & -0.710029 \\ 26 & 6 & -1.780201 & 0.718887 & 1.159148 \\ 27 & 6 & -2.795260 & 0.532457 & 0.052696 \\ 28 & 1 & -0.232745 & -0.373923 & 2.222284 \\ 29 & 1 & -1.754539 & -1.229245 & 2.063366 \\ 30 & 1 & -2.278961 & 1.110828 & 2.054963 \\ 31 & 1 & -1.060355 & 1.504515 & 0.869034 \\ 32 & 1 & -3.543928 & -0.701674 & -1.474456 \\ 33 & 1 & -1.450899 & -1.981686 & -1.602716 \\ 34 & 1 & -2.265623 & -2.586288 & -0.187657 \\ 35 & 6 & -3.814584 & 1.628699 & -0.119015 \\ 36 & 1 & -4.478402 & 1.436363 & -0.965733 \\ 37 & 1 & -4.432395 & 1.734395 & 0.781724 \\ 38 & 1 & -3.330890 & 2.601498 & -0.280967 \\ 39 & 1 & 2.000934 & 1.225373 & 1.533583 \\ 40 & 1 & 3.492746 & 1.646498 & 0.803041 \\ --------------------------------------------------- \\ -\end{array}$

6) TS Cb-to-Cc

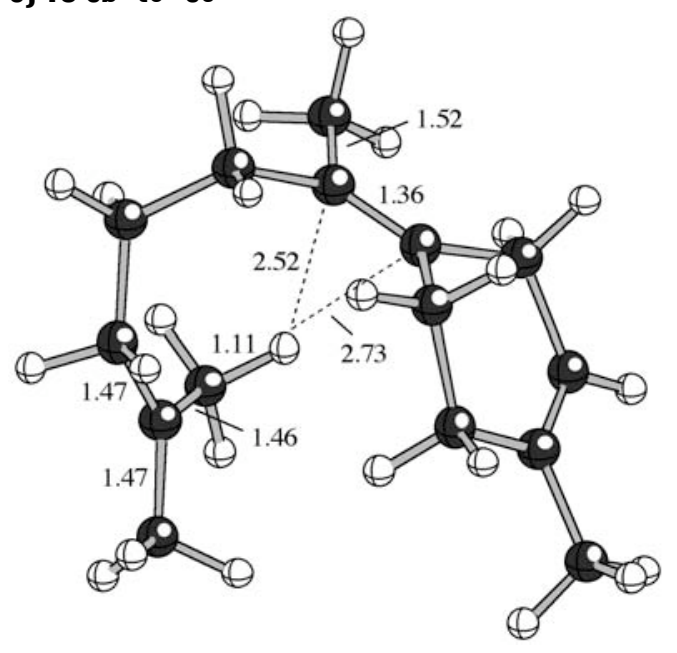


B3LYP/6-31+G[d,p]: -586.3991759 hartrees [-367965.48287725 kcal/mol]

Imaginary frequencies: $1[-115.0768 \mathrm{1} / \mathrm{cm}]$

Zero-point correction $\mathbf{0} 0.361134$ (Hartree/Particle)

mPW1PW91/6-31+G[d,p]//B3LYP/6-31+G[d,p]: -586.2618644 hartrees [-367879.319911 kcal/mol]

Coordinates:

\begin{tabular}{|c|c|c|c|c|}
\hline Center & fitomic & & ordinates [AI & Ingstroms] \\
\hline number & - number & $x$ & $\{\quad Y$ & Z \\
\hline 1 & 6 & 2.685394 & -0.679494 & 0.768997 \\
\hline 2 & 6 & 2.786759 & 0.391316 & -0.033549 \\
\hline 3 & 6 & 1.830562 & 0.582630 & -1.190930 \\
\hline 4 & 6 & 1.026377 & -0.688563 & -1.526561 \\
\hline 5 & 6 & 0.495078 & -1.377340 & -0.284909 \\
\hline 6 & 6 & 1.629562 & -1.757472 & 0.649711 \\
\hline 7 & 1 & 1.149007 & 1.423126 & -0.970358 \\
\hline 8 & 1 & 3.410638 & -0.813522 & 1.569960 \\
\hline 9 & 1 & 0.247938 & -0.451715 & -2.254694 \\
\hline 10 & 1 & 1.707917 & -1.391861 & -2.025944 \\
\hline 11 & 1 & 2.390300 & 0.901623 & -2.080066 \\
\hline 12 & 6 & -1.937828 & -1.367445 & $5-1.023324$ \\
\hline 13 & 6 & -2.958768 & -0.292787 & $7-0.572989$ \\
\hline 14 & 1 & -2.511465 & -2.291364 & $4-1.173432$ \\
\hline 15 & 1 & -1.561126 & -1.092056 & $6-2.011712$ \\
\hline 16 & 6 & -2.461962 & 1.160134 & -0.645253 \\
\hline 17 & 1 & -3.823203 & -0.353452 & $2-1.242249$ \\
\hline 18 & 1 & -3.348423 & -0.514897 & $7 \quad 0.426306$ \\
\hline 19 & 6 & -1.698543 & 1.791668 & 0.440755 \\
\hline 20 & 6 & -0.805917 & -1.677803 & $3-0.050964$ \\
\hline 21 & 6 & -1.461438 & 1.130472 & 1.715661 \\
\hline 22 & 1 & -1.053108 & 1.777093 & 2.492162 \\
\hline 23 & 1 & -2.328831 & 0.559632 & 2.063436 \\
\hline 24 & 1 & -0.719559 & 0.340937 & 1.452670 \\
\hline 25 & 6 & -1.167248 & 3.152018 & 0.237955 \\
\hline 26 & 1 & -1.288390 & 3.534619 & -0.775611 \\
\hline 27 & 1 & -1.674174 & 3.820945 & 0.952690 \\
\hline 28 & 1 & -0.111024 & 3.182899 & 0.539715 \\
\hline 29 & 1 & -3.326191 & 1.857933 & -0.723968 \\
\hline 30 & 1 & -1.933791 & 1.370281 & -1.590098 \\
\hline 31 & 6 & -1.276151 & -2.496987 & $7 \quad 1.138192$ \\
\hline 32 & 1 & -1.427580 & -3.541272 & $2 \quad 0.834453$ \\
\hline 33 & 1 & -0.581271 & -2.505724 & $4 \quad 1.978622$ \\
\hline 34 & 1 & -2.245779 & -2.150565 & $\begin{array}{ll}5 & 1.515703\end{array}$ \\
\hline 35 & 1 & 2.101754 & -2.682651 & 0.278006 \\
\hline 36 & 1 & 1.269417 & -2.009711 & 1.650752 \\
\hline 37 & 6 & 3.855527 & 1.439926 & 0.138851 \\
\hline
\end{tabular}




\begin{tabular}{lllll}
38 & 1 & 4.514922 & 1.476039 & -0.737631 \\
39 & 1 & 3.420257 & 2.443470 & 0.241315 \\
40 & 1 & 4.473333 & 1.248543 & 1.020123 \\
\hline
\end{tabular}

IRC: TS Cb-to-Cc $\rightarrow$ Cb
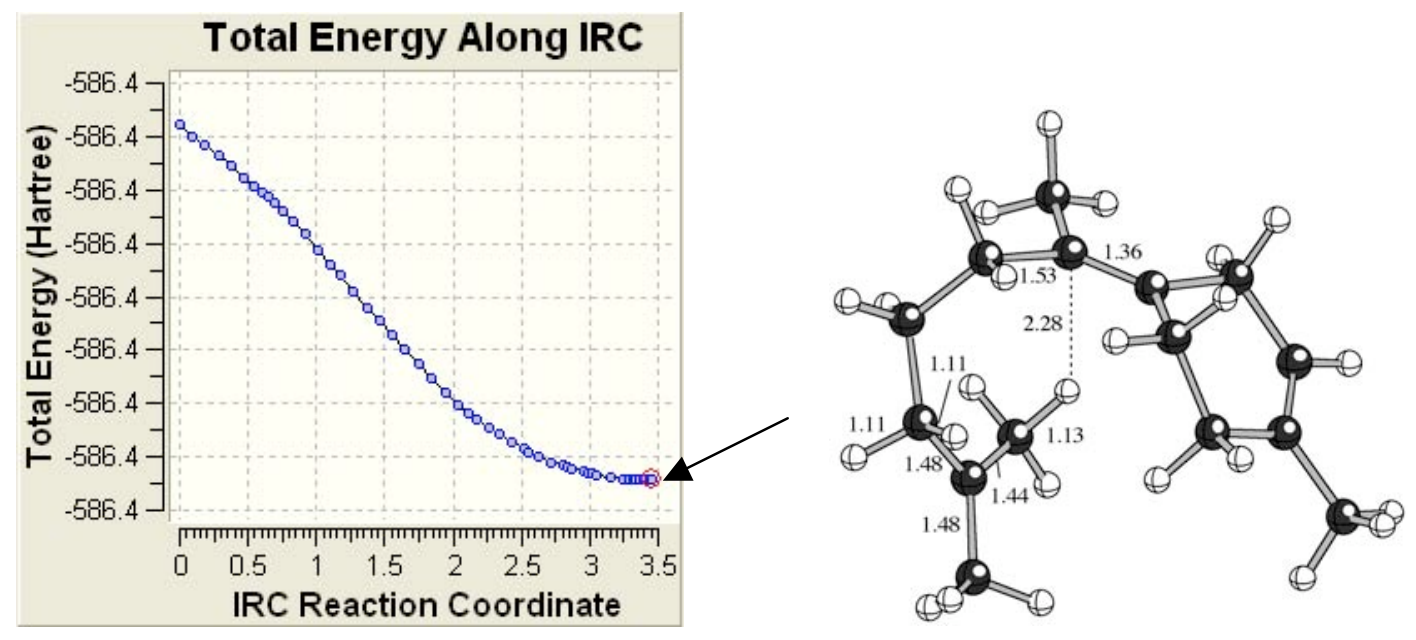

IRC: TS Cb-to-Cc $\rightarrow$ Cb

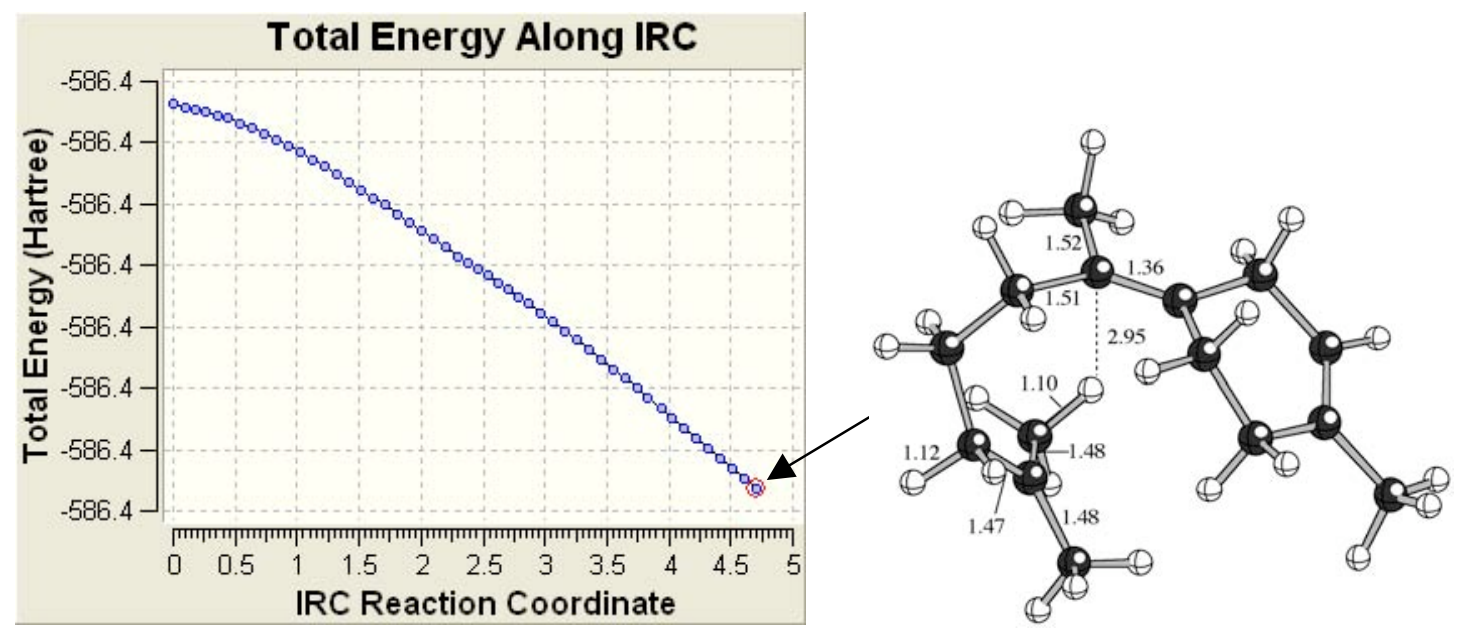

7) CC 


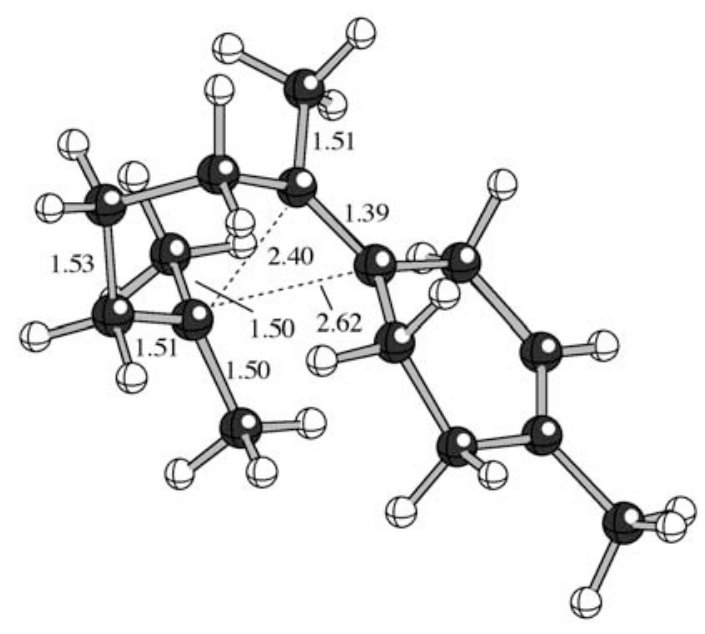

B3LYP/6-31+G[d,p]: -586.4148545 hartrees [-367975.32119875 kcal/mol]

Imaginary frequencies: none

Zero-point correction = 0.364943 (Hartree/Particle $)$

mPW1PU91/6-31+G[d,p]//B3LYP/6-31+G[d,p]: -586.2810515 hartrees [-367891.35981625 kcal/mol]

Coordinates:

\begin{tabular}{|c|c|c|c|c|}
\hline \multirow{2}{*}{$\begin{array}{l}\text { Center } \\
\text { number }\end{array}$} & Atomic & \multicolumn{3}{|c|}{ Coordinates [Angstroms] } \\
\hline & number & $x$ & $Y$ & Z \\
\hline 1 & 6 & 0.200341 & -0.753922 & -0.200616 \\
\hline 2 & 6 & -1.709192 & -2.046143 & 0.828698 \\
\hline 3 & 1 & -2.799625 & -1.996338 & 0.872597 \\
\hline 4 & 1 & -1.454437 & -3.071446 & 0.526658 \\
\hline 5 & 1 & -1.314746 & -1.902890 & 1.834449 \\
\hline 6 & 6 & -1.147713 & -1.102311 & -0.213203 \\
\hline 7 & 6 & -2.045777 & -0.913785 & -1.416932 \\
\hline 8 & 6 & -1.439787 & 1.133862 & 0.592609 \\
\hline 9 & 6 & -3.074185 & 0.201099 & -1.127574 \\
\hline 10 & 1 & -2.566158 & -1.856005 & $5-1.621750$ \\
\hline 11 & 1 & -1.483201 & -0.661584 & $4-2.317370$ \\
\hline 12 & 6 & -2.298659 & 1.415465 & -0.610837 \\
\hline 13 & 1 & -3.632667 & 0.459149 & -2.031613 \\
\hline 14 & 1 & -3.810255 & -0.133525 & $5-0.389174$ \\
\hline 15 & 6 & -0.231226 & 2.007915 & 0.770964 \\
\hline 16 & 1 & -0.603122 & 2.971426 & 1.153880 \\
\hline 17 & 1 & 0.472317 & 1.627410 & 1.514825 \\
\hline 18 & 1 & 0.286921 & 2.217047 & -0.163457 \\
\hline 19 & 6 & -2.118024 & 0.765684 & 1.877823 \\
\hline 20 & 1 & -1.434442 & 0.300140 & 2.592625 \\
\hline 21 & 1 & -2.436965 & 1.712982 & 2.339071 \\
\hline 22 & 1 & -3.005280 & 0.146302 & 1.757006 \\
\hline 23 & 6 & 1.070077 & -1.165792 & 0.964132 \\
\hline 24 & 6 & 0.923197 & -0.240832 & -1.423317 \\
\hline
\end{tabular}




$\begin{array}{lllll}25 & 6 & 2.511125 & -0.729147 & 0.918945 \\ 26 & 6 & 2.241944 & 0.524249 & -1.200957 \\ 27 & 6 & 3.063791 & 0.027070 & -0.037711 \\ 28 & 1 & 0.264192 & 0.330608 & -2.079824 \\ 29 & 1 & 1.162564 & -1.161150 & -1.983006 \\ 30 & 1 & 2.830560 & 0.452772 & -2.123292 \\ 31 & 1 & 2.055761 & 1.599129 & -1.072892 \\ 32 & 1 & 3.121035 & -1.075106 & 1.750524 \\ 33 & 1 & 0.612201 & -0.831849 & 1.907542 \\ 34 & 1 & 1.024004 & -2.265774 & 1.032390 \\ 35 & 6 & 4.509864 & 0.444487 & -0.012639 \\ 36 & 1 & 5.017875 & 0.092808 & 0.888623 \\ 37 & 1 & 5.045732 & 0.049234 & -0.884512 \\ 38 & 1 & 4.604453 & 1.537066 & -0.054112 \\ 39 & 1 & -1.690094 & 1.848469 & -1.411222 \\ 40 & 1 & -3.001282 & 2.203739 & -0.294279 \\ ------------------------------------~\end{array}$

8] TS Cc-to-cuprenyl-I

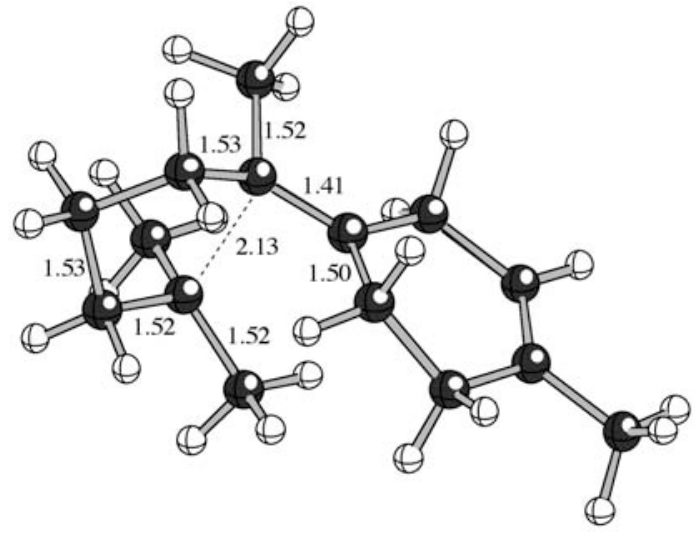

B3LYP/6-31+G[d,p]: -586.4144347 hartrees [-367975.05777425 kcal/mol]

Imaginary frequencies: $1[-113.52641 / \mathrm{cm}]$

Zero-point correction = 0.365337 (Hartree/Particle)

mPW1PW91/6-31+G[d,p]//B3LYP/6-31+G[d,p]: -586.2824575 hartrees [-367892.24208125 kcal/mol]

Coordinates:

\begin{tabular}{|c|c|c|c|c|}
\hline \multirow{2}{*}{$\begin{array}{l}\text { Center } \\
\text { number }\end{array}$} & \multirow{2}{*}{$\begin{array}{l}\text { Atomic } \\
\text { number }\end{array}$} & \multicolumn{3}{|c|}{ Coordinates [fingstroms] } \\
\hline & & r & $Y$ & Z \\
\hline 1 & 6 & -0.249180 & 0.625432 & -0.380880 \\
\hline 2 & 6 & 1.660897 & 2.143116 & 0.249125 \\
\hline 3 & 1 & 2.751839 & 2.146928 & 0.280637 \\
\hline 4 & 1 & 1.359095 & 3.006121 & -0.358009 \\
\hline 5 & 1 & 1.289923 & 2.309793 & 1.259448 \\
\hline 6 & 6 & 1.133804 & 0.884808 & -0.429643 \\
\hline 7 & 6 & 1.998386 & 0.460824 & -1.616331 \\
\hline
\end{tabular}




$\begin{array}{ccccc}8 & 6 & 1.510209 & -0.791048 & 0.821527 \\ 9 & 6 & 3.106181 & -0.476792 & -1.105543 \\ 10 & 1 & 2.418734 & 1.358511 & -2.081999 \\ 11 & 1 & 1.420414 & -0.050789 & -2.388362 \\ 12 & 6 & 2.388455 & -1.483844 & -0.209097 \\ 13 & 1 & 3.626817 & -0.970650 & -1.930305 \\ 14 & 1 & 3.862047 & 0.081598 & -0.542394 \\ 15 & 6 & 0.330151 & -1.619956 & 1.291654 \\ 16 & 1 & 0.745251 & -2.436423 & 1.898597 \\ 17 & 1 & -0.359057 & -1.068551 & 1.935270 \\ 18 & 1 & -0.220023 & -2.084550 & 0.473996 \\ 19 & 6 & 2.235216 & -0.145333 & 1.979833 \\ 20 & 1 & 1.582912 & 0.485484 & 2.588789 \\ 21 & 1 & 2.564381 & -0.966553 & 2.631837 \\ 22 & 1 & 3.121834 & 0.418389 & 1.692319 \\ 23 & 6 & -1.116835 & 1.363046 & 0.597540 \\ 24 & 6 & -0.960960 & -0.218018 & -1.394918 \\ 25 & 6 & -2.562461 & 0.948666 & 0.674999 \\ 26 & 6 & -2.285045 & -0.881804 & -0.969458 \\ 27 & 6 & -3.113327 & -0.058114 & -0.014012 \\ 28 & 1 & -0.297239 & -0.945748 & -1.864404 \\ 29 & 1 & -1.190487 & 0.517435 & -2.188857 \\ 30 & 1 & -2.863569 & -1.087067 & -1.877597 \\ 31 & 1 & -2.097368 & -1.868741 & -0.525700 \\ 32 & 1 & -3.171444 & 1.531465 & 1.361837 \\ 33 & 1 & -0.656139 & 1.331201 & 1.595874 \\ 34 & 1 & -1.046288 & 2.431498 & 0.325337 \\ 35 & 6 & -4.559822 & -0.448459 & 0.125695 \\ 36 & 1 & -5.074580 & 0.162458 & 0.870967 \\ 37 & 1 & -5.087399 & -0.341493 & -0.830099 \\ 38 & 1 & -4.654630 & -1.500907 & 0.421654 \\ 39 & 1 & 1.781940 & -2.162651 & -0.818726 \\ 40 & 1 & 3.099032 & -2.111909 & 0.346936 \\ -----------------------------------------------------\end{array}$

IRC: TS [CC-to-cuprenyl-I] $\rightarrow$ CC 


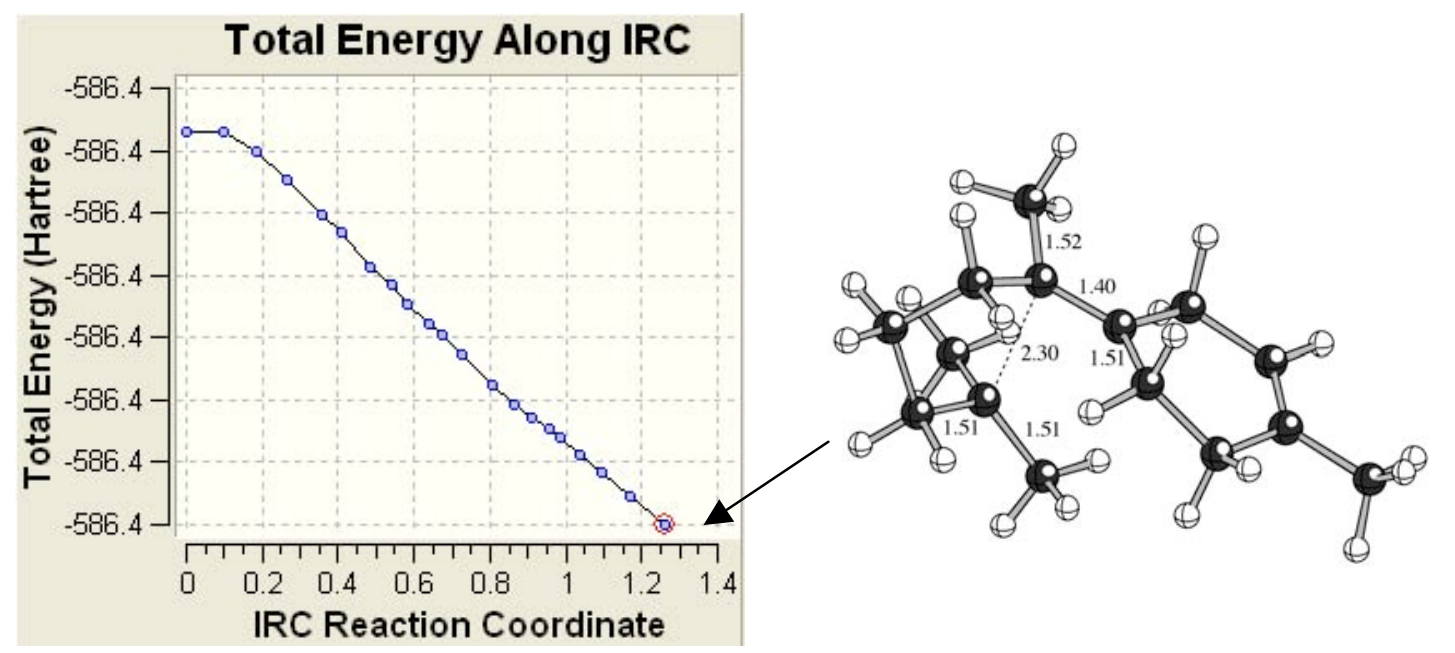

IRC: TS [Cc-to-cuprenyl-I] -> cuprenyl-I

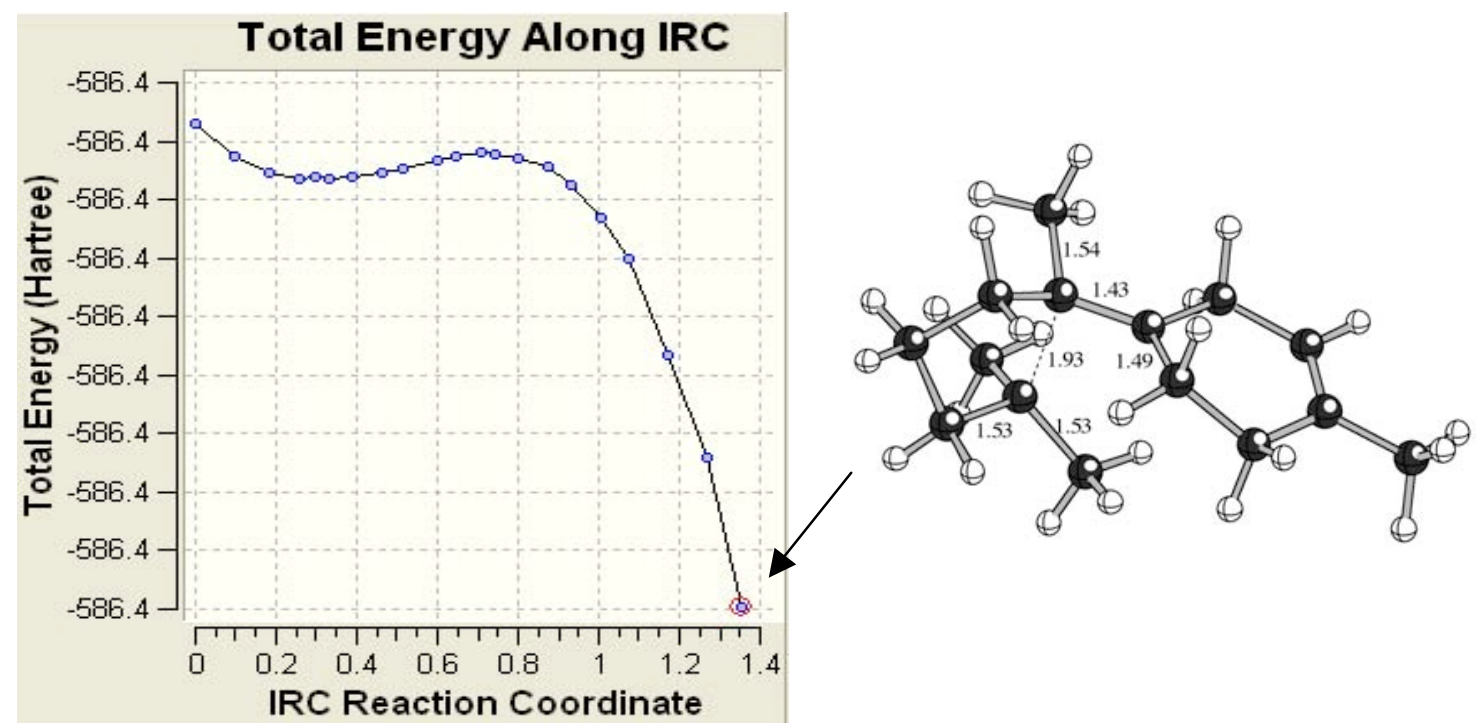

Although it looks in this image like there is a minimum between the IS and cuprenyl-I, no true minimum could be found. Optimizations starting from points in the apparent minimum did not converge, presumably since the surface in this area is flat. Optimizations starting from the hilltop led to cuprenyl-I. Mote that the energy difference between the apparent minimum and the hilltop is only $0.014 \mathrm{kcal} / \mathrm{mol}$. Mote also that for the TS, animation of the vibrational mode corresponding to the imaginary frequency clearly indicates $C---C$ bond formation. 
9) Cuprenyl-I

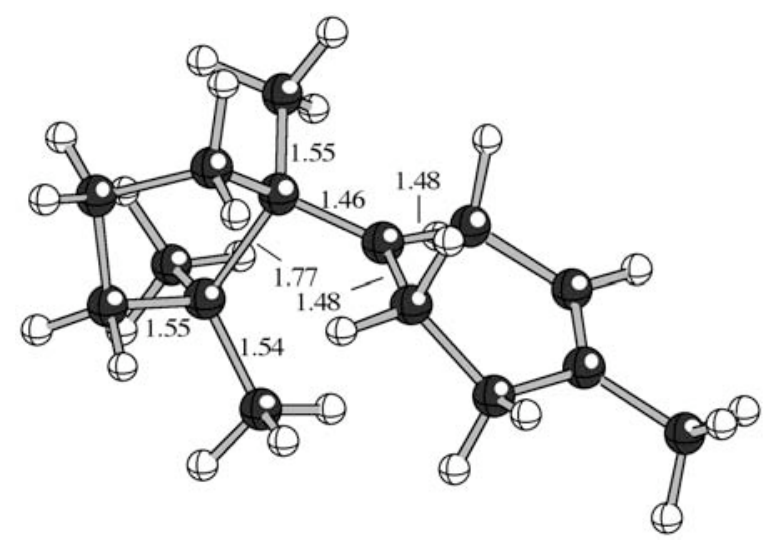

B3LYP/6-31+G[d,p]: -586.4157555 hartrees [-367975.88657625 kcal/mol] Imaginary frequencies: none

Zero-point correction = 0.365941 (Hartree/Particle $)$

mPU1PW91/6-31+G[d,p]//B3LYP/6-31+G[d,p]: -586.2864098 hartrees [-367894.7221495 kcal/mol]

Coordinates:

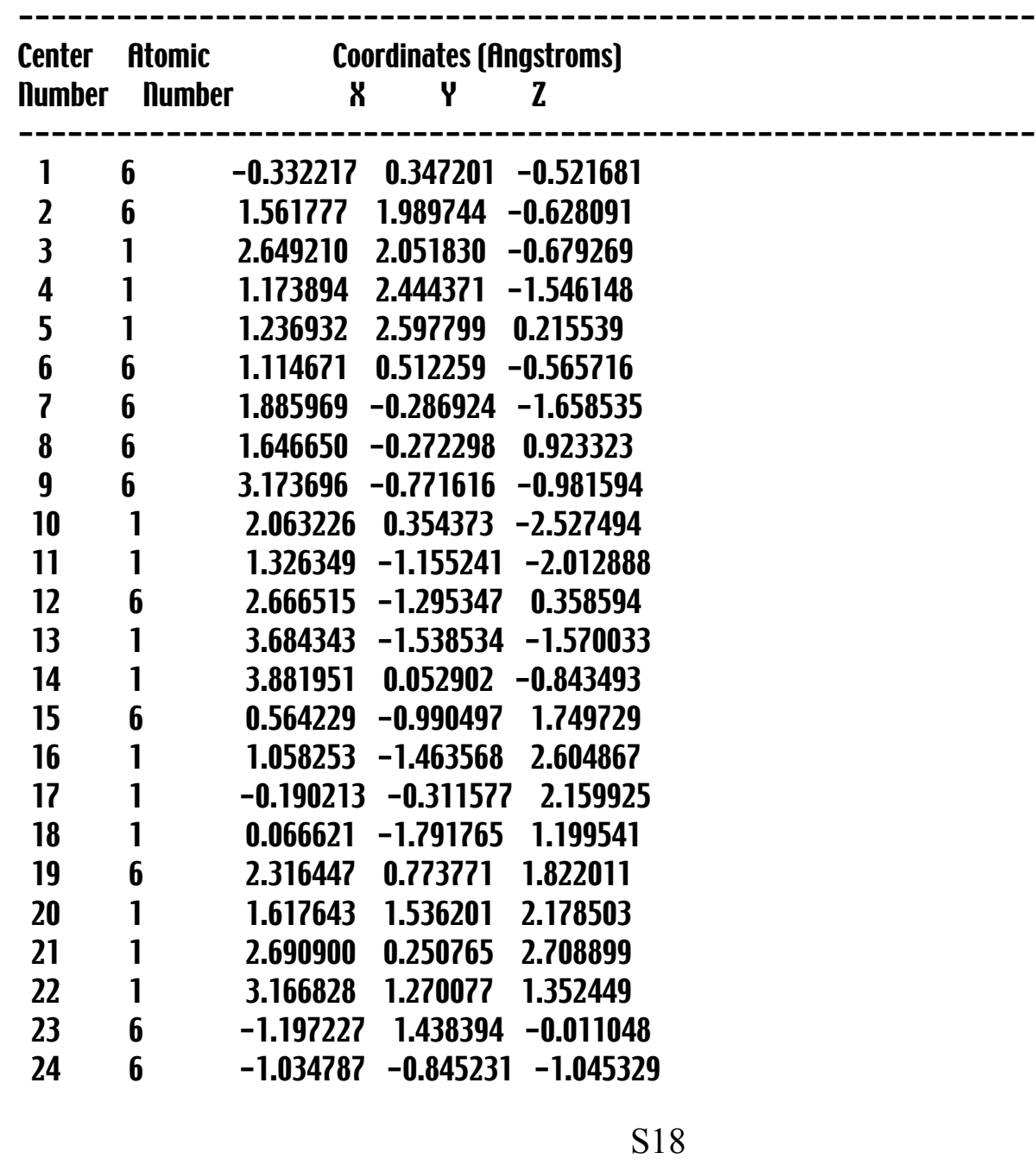




\begin{tabular}{|c|c|c|c|c|}
\hline 25 & 6 & -2.662580 & 1.147682 & 0.167596 \\
\hline 26 & 6 & -2.350412 & -1.246212 & -0.349294 \\
\hline 27 & 6 & -3.211520 & -0.066701 & 0.036307 \\
\hline 28 & 1 & -0.372471 & -1.699427 & -1.188270 \\
\hline 29 & 1 & -1.285844 & -0.496321 & -2.071215 \\
\hline 30 & 1 & -2.898157 & -1.908454 & -1.028187 \\
\hline 31 & 1 & -2.134672 & -1.851516 & 0.541187 \\
\hline 32 & 1 & -3.273961 & 1.999865 & 0.451981 \\
\hline 33 & 1 & -0.748255 & 1.839507 & 0.912118 \\
\hline 34 & 1 & -1.047652 & 2.277338 & -0.718007 \\
\hline 35 & 6 & -4.667436 & -0.343503 & 0.287410 \\
\hline 36 & 1 & -5.199588 & 0.552447 & 0.615095 \\
\hline 37 & 1 & -5.155550 & -0.723513 & -0.618244 \\
\hline 38 & 1 & -4.788456 & -1.114707 & 1.058572 \\
\hline 39 & 1 & 2.172672 & -2.262936 & 0.208970 \\
\hline 40 & 1 & 3.463344 & -1.454255 & 1.093512 \\
\hline
\end{tabular}

10) TS bisabolyl-I-to-bisabolyl-II

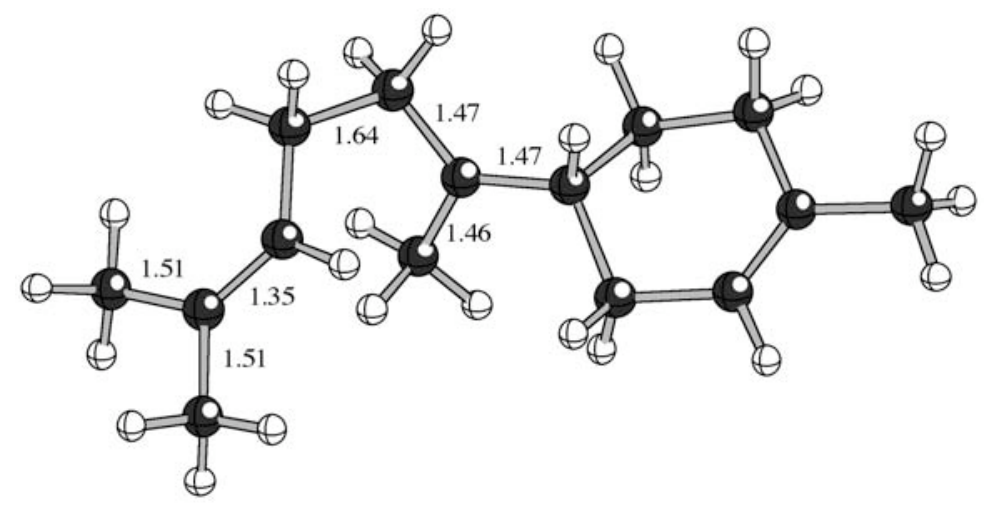

B3LYP/6-31+G[d,p]: -586.4074559 hartrees [-367970.67857725 kcal $/ \mathrm{mol}]$ Imaginary frequencies: 1 [-97.7772 1/cm]

Zero-point correction $=\mathbf{0 . 3 6 2 5 4 4}$ (Hartree/Particle)

mPW1PW91/6-31+G[d,p]//B3LYP/6-31+G[d,p]: -586.2680972 hartrees [-367883.230993 kcal/mol] Coordinates:

\begin{tabular}{lllll}
\multicolumn{2}{c}{$\begin{array}{l}\text { Center } \\
\text { number }\end{array}$} & \multicolumn{2}{c}{ Atomic } & \multicolumn{3}{c}{ Coordinates [fingstroms] } \\
\hline 1 & 6 & 2.862342 & 1.465698 & 0.086356 \\
2 & 6 & 3.832733 & 0.567978 & -0.141967 \\
3 & 6 & 3.559641 & -0.919400 & -0.100194 \\
4 & 6 & 2.203769 & -1.297618 & 0.509948 \\
5 & 6 & 1.094069 & -0.313974 & -0.077507 \\
6 & 6 & 1.423292 & 1.121447 & 0.382786
\end{tabular}




$\begin{array}{ccccc}7 & 1 & 3.651986 & -1.338308 & -1.112281 \\ 8 & 1 & 3.097635 & 2.527007 & 0.050086 \\ 9 & 1 & 1.966997 & -2.342354 & 0.288232 \\ 10 & 1 & 2.219478 & -1.176606 & 1.597779 \\ 11 & 1 & 4.335668 & -1.417902 & 0.496931 \\ 12 & 6 & -0.899971 & -1.802934 & -0.561940 \\ 13 & 6 & -2.324988 & -1.212406 & -1.118448 \\ 14 & 1 & -1.174090 & -2.710901 & -0.013365 \\ 15 & 1 & -0.280440 & -2.074949 & -1.417282 \\ 16 & 6 & -2.675275 & 0.214641 & -0.848559 \\ 17 & 1 & -2.237834 & -1.361040 & -2.199091 \\ 18 & 1 & -3.100318 & -1.886833 & -0.758215 \\ 19 & 6 & -3.767147 & 0.677185 & -0.197252 \\ 20 & 6 & -0.182013 & -0.899879 & 0.340562 \\ 21 & 6 & -4.047084 & 2.158563 & -0.139803 \\ 22 & 1 & -5.002277 & 2.383047 & -0.630251 \\ 23 & 1 & -4.145925 & 2.500060 & 0.897888 \\ 24 & 1 & -3.268311 & 2.748833 & -0.629301 \\ 25 & 6 & -4.809461 & -0.184800 & 0.468697 \\ 26 & 1 & -5.768331 & -0.083153 & -0.054524 \\ 27 & 1 & -4.560354 & -1.247117 & 0.499964 \\ 28 & 1 & -4.983889 & 0.155157 & 1.496336 \\ 29 & 1 & -2.015027 & 0.956051 & -1.301693 \\ 30 & 1 & 1.182346 & -0.382334 & -1.168342 \\ 31 & 6 & -0.801179 & -0.572539 & 1.625238 \\ 32 & 1 & -0.124156 & -0.154236 & 2.369422 \\ 33 & 1 & -1.535144 & 0.221110 & 1.345450 \\ 34 & 1 & -1.401568 & -1.388180 & 2.035379 \\ 35 & 1 & 1.217153 & 1.248519 & 1.453320 \\ 36 & 1 & 0.761369 & 1.822742 & -0.140320 \\ 37 & 6 & 5.247444 & 0.967260 & -0.468221 \\ 38 & 1 & 5.945885 & 0.592413 & 0.290169 \\ 39 & 1 & 5.564716 & 0.540767 & -1.428262 \\ 40 & 1 & 5.357415 & 2.052859 & -0.526194 \\ ----------------------------------------------------- \\ \end{array}$

IRC: TS(bisabolyl-I-to-bisabolyl-II) -> bisabolyl-I 


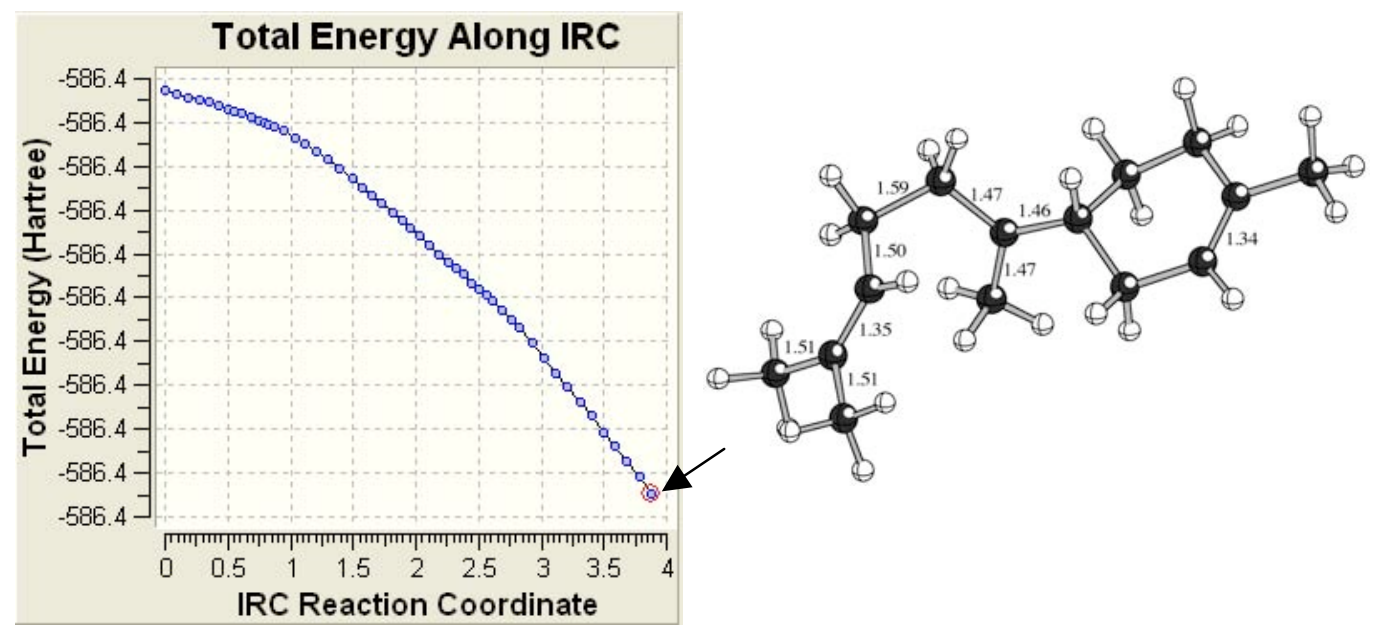

IRC: TS[bisabolyl-I-to-bisabolyl-II] -> bisabolyl-II

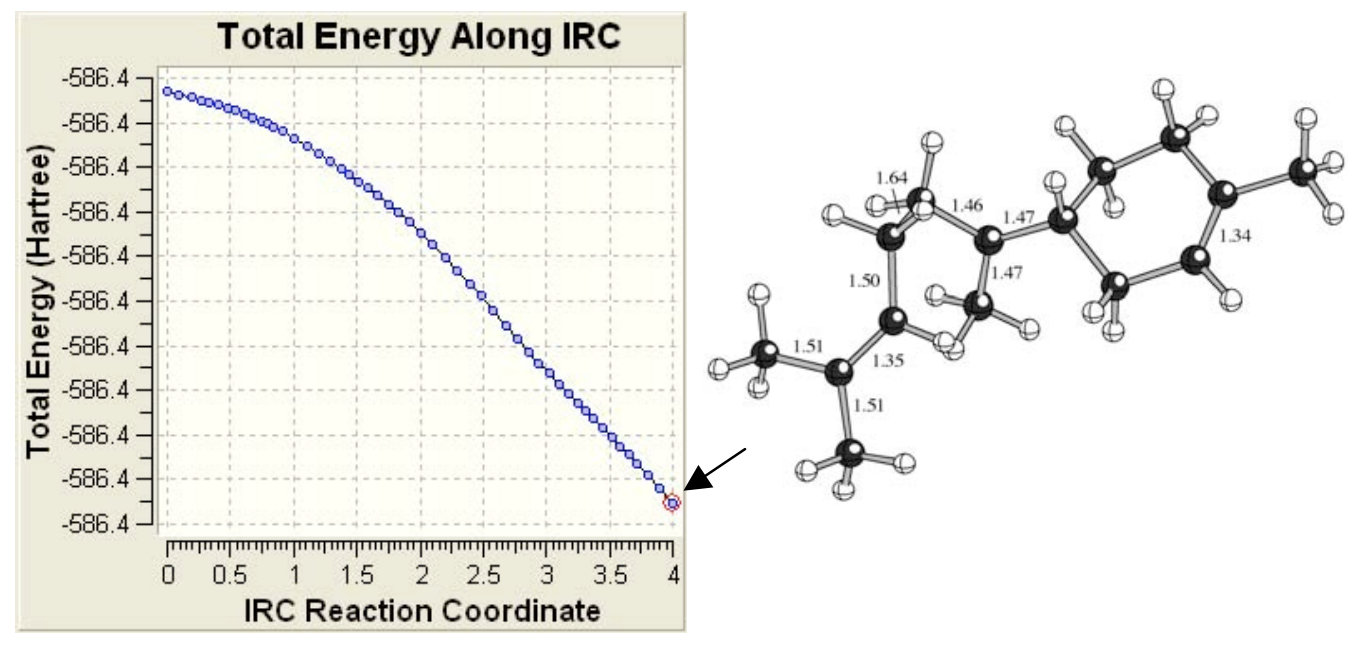

11] Bisabolyl-II:

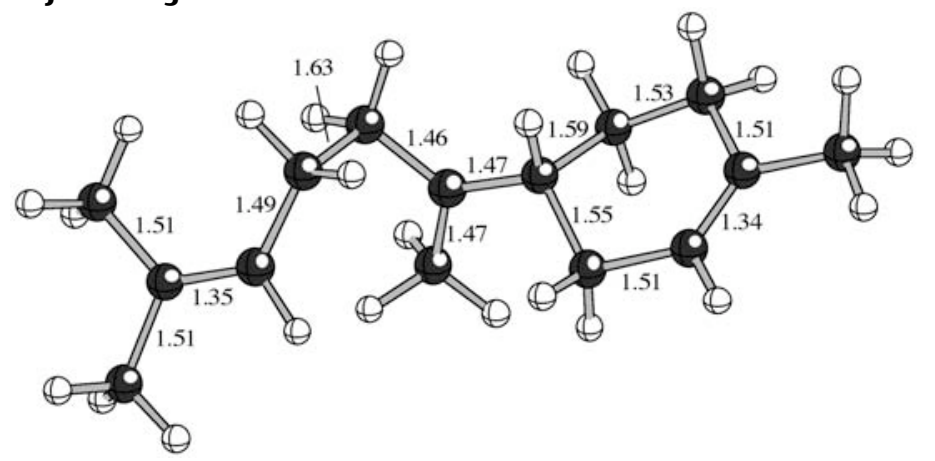

B3LYP/6-31+G[d,p]: -586.4157364 hartrees [-367975.874591 kcal/mol]

Imaginary frequencies: none

Zero-point correction $=0.363176$ (Hartree/Particle $)$

mPW1PW91/6-31+G[d,p]//B3LYP/6-31+G[d,p]: -586.2760635 hartrees [-367888.22984625 kcal/mol] 
Coordinates:

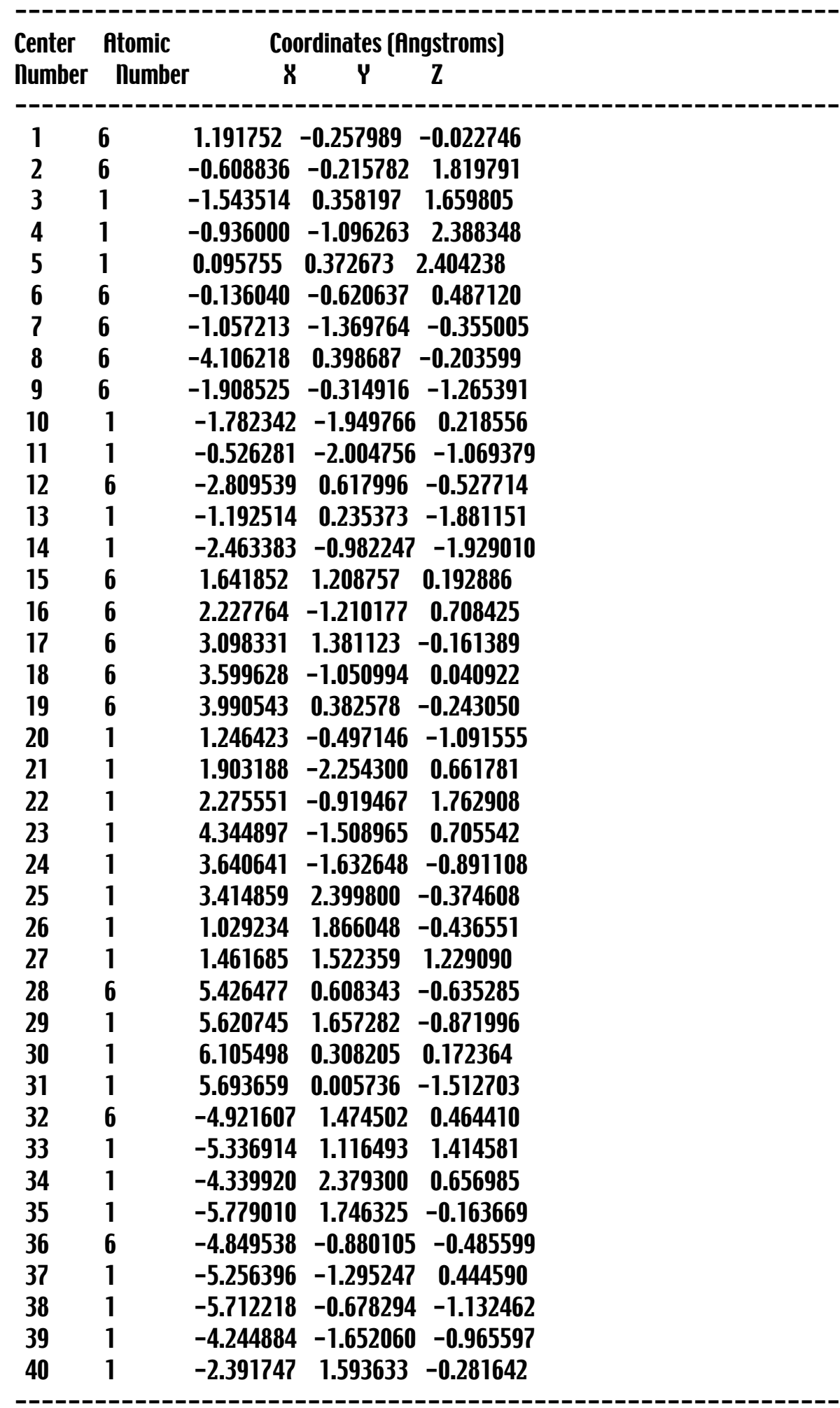

12) TS bisabolyl-II-to-B 


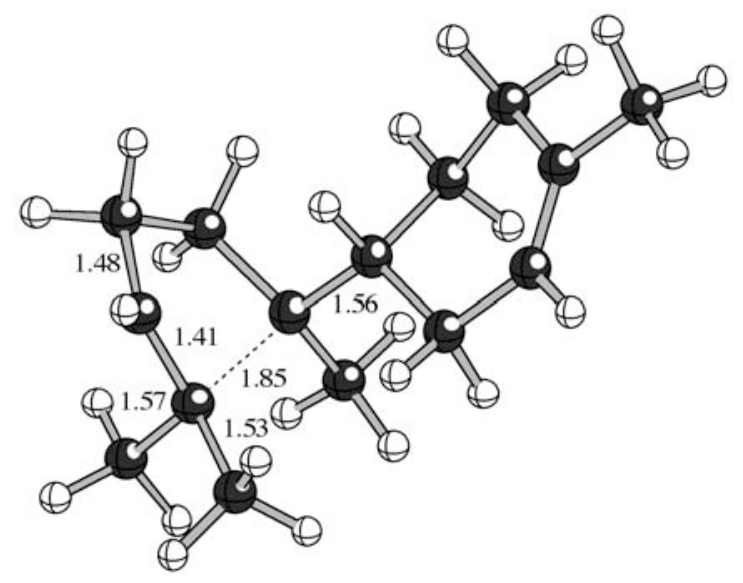

B3LYP/6-31+G[d,p]: -586.3762553 hartrees [-367951.10020075 kcal/mol]

Imaginary frequencies: $1[-343.5780 \mathrm{1} / \mathrm{cm}]$

Zero-point correction $\mathbf{0} 0.364926$ (Hartree/Particle)

mPW1PW91/6-31+G[d,p]//B3LYP/6-31+G[d,p]: -586.245441 hartrees [-367869.0142275 kcal/mol]

Coordinates:

\begin{tabular}{ccccc}
\hline Center & fitomic & \multicolumn{3}{c}{ Coordinates [fingstroms] } \\
number & number & X & $Y$ & Z \\
-1 & 6 & 0.431135 & -0.182510 & 0.062263 \\
2 & 6 & -1.235507 & -0.284191 & 2.014827 \\
3 & 1 & -2.241088 & -0.546441 & 2.342898 \\
4 & 1 & -0.550365 & -0.979996 & 2.512831 \\
5 & 1 & -0.991384 & 0.717710 & 2.377228 \\
6 & 6 & -1.038559 & -0.433575 & 0.515604 \\
7 & 6 & -1.583800 & -1.768629 & -0.080097 \\
8 & 6 & -2.257720 & 0.695836 & -0.292908 \\
9 & 6 & -1.976478 & -1.423543 & -1.531557 \\
10 & 1 & -2.432276 & -2.136536 & 0.498107 \\
11 & 1 & -0.828150 & -2.554761 & -0.049509 \\
12 & 6 & -2.073950 & 0.049934 & -1.536801 \\
13 & 1 & -1.236407 & -1.744181 & -2.274507 \\
14 & 1 & -2.925192 & -1.869514 & -1.878123 \\
15 & 6 & 1.015960 & 1.228749 & 0.292215 \\
16 & 6 & 1.402886 & -1.192504 & 0.733821 \\
17 & 6 & 2.495867 & 1.291452 & -0.016261 \\
18 & 6 & 2.763814 & -1.174681 & 0.025682 \\
19 & 6 & 3.297614 & 0.227365 & -0.168896 \\
20 & 1 & 0.482706 & -0.372258 & -1.021091 \\
21 & 1 & 1.007179 & -2.212830 & 0.736406 \\
22 & 1 & 1.551372 & -0.904643 & 1.780711 \\
23 & 1 & 3.479977 & -1.759833 & 0.616958 \\
24 & 1 & 2.701654 & -1.686428 & -0.947117 \\
25 & 1 & 2.908755 & 2.291506 & -0.134423
\end{tabular}




\begin{tabular}{|c|c|c|c|}
\hline 26 & 1 & 0.514221 & $1.962420-0.342850$ \\
\hline 27 & 1 & 0.837240 & $1.551986 \quad 1.328314$ \\
\hline 28 & 6 & 4.754250 & $0.349650-0.529909$ \\
\hline 29 & 1 & 5.048132 & $1.390778-0.686053$ \\
\hline 30 & 1 & 5.390609 & $-0.071886 \quad 0.258320$ \\
\hline 31 & 1 & 4.979770 & $-0.208979-1.447526$ \\
\hline 32 & 6 & -2.034428 & $2.207150-0.253632$ \\
\hline 33 & 1 & -1.679382 & $2.518659 \quad 0.732187$ \\
\hline 34 & 1 & -1.316584 & $2.552156-0.999097$ \\
\hline 35 & 1 & -2.981290 & $2.726451-0.432479$ \\
\hline 36 & 6 & -3.624771 & $0.367822 \quad 0.397627$ \\
\hline 37 & 1 & -3.629593 & 0.8301791 .385490 \\
\hline 38 & 1 & -4.414282 & $0.834569-0.196708$ \\
\hline 39 & 1 & -3.847352 & $-0.692370 \quad 0.492995$ \\
\hline 40 & 1 & -1.846143 & $0.614292-2.444836$ \\
\hline
\end{tabular}

IRC: TS(bisabolyl-II-to-B) -> bisabolyl-II

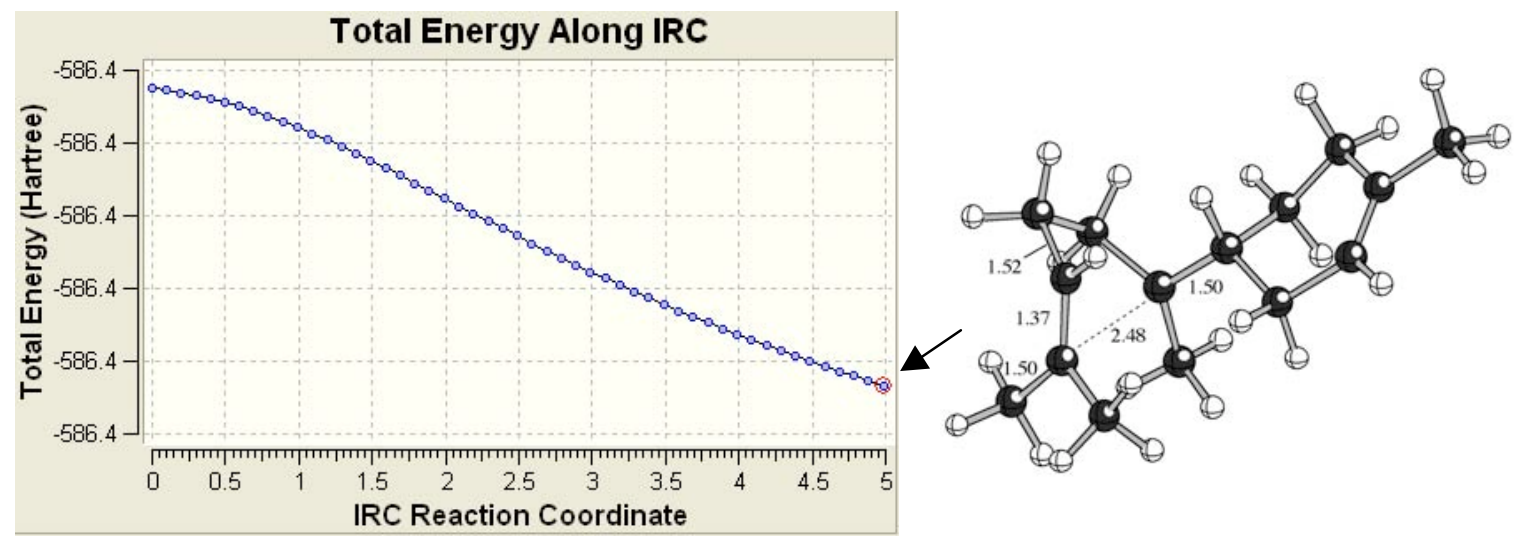

IRC: TS[bisabolyl-II-to-B] -> B
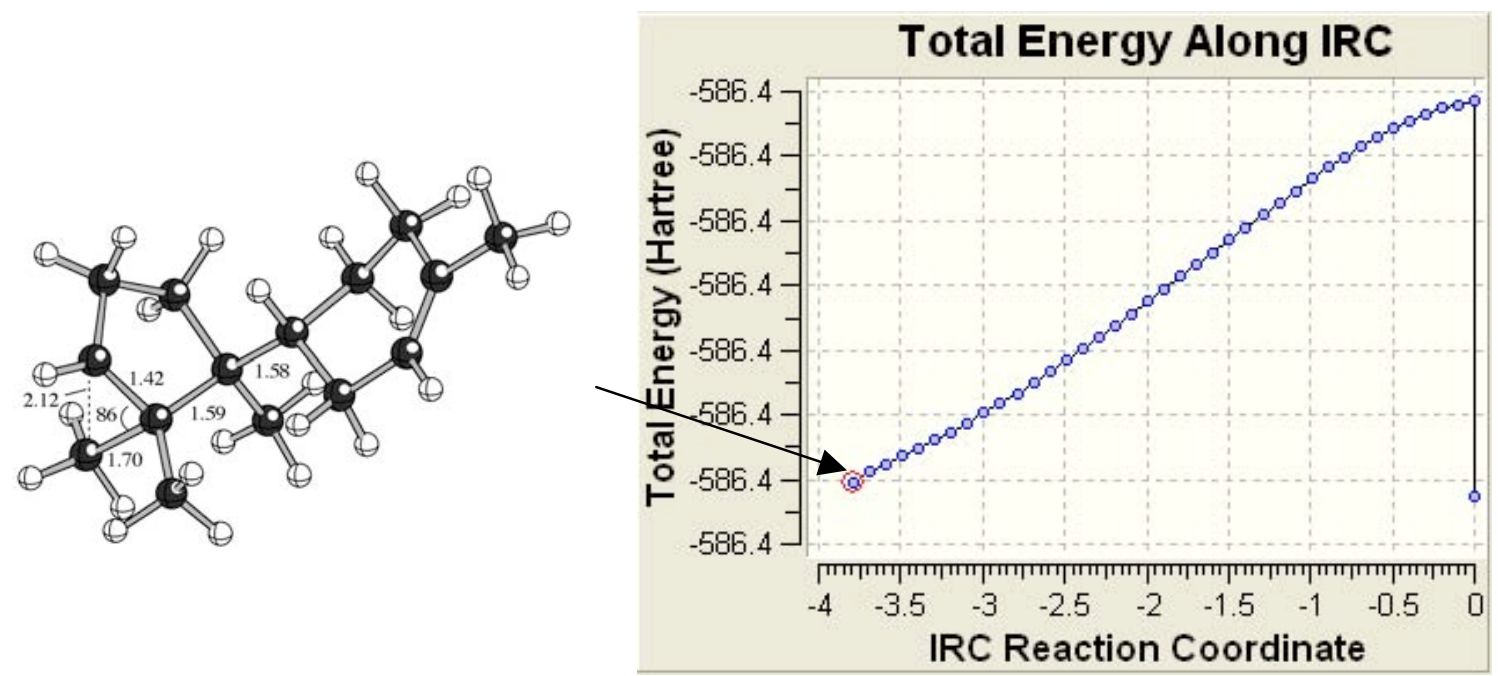
energy of endpoint shown: B3LYP/6-31+G[d,p]: -586.38811518 hartrees

13) B

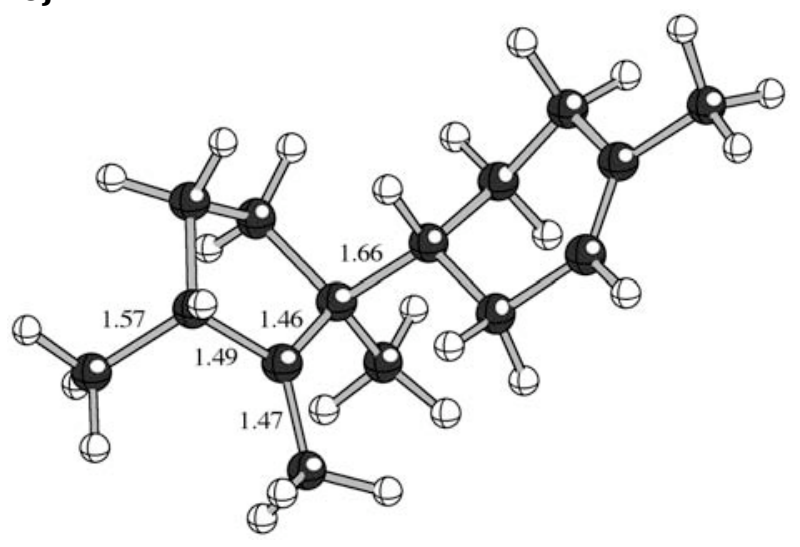

B3LYP/6-31+G[d,p]: -586.4236978 hartrees [-367980.8703695 kcal/mol] Imaginary frequencies: none Zero-point correction = 0.366143 (Hartree/Particle)

mPW1PU91/6-31+G[d,p]//B3LYP/6-31+G[d,p]:-586.2942943 hartrees [-367899.66967325 kcal/mol] Coordinates:

\begin{tabular}{|c|c|c|c|c|}
\hline \multirow{2}{*}{$\begin{array}{l}\text { Center } \\
\text { number }\end{array}$} & \multirow{2}{*}{$\begin{array}{l}\text { Atomic } \\
\text { number }\end{array}$} & \multicolumn{3}{|c|}{ Coordinates [fingstroms] } \\
\hline & & X & $Y$ & $\mathbf{Z}$ \\
\hline 1 & 6 & 0.502222 & -0.170589 & 0.080975 \\
\hline 2 & 6 & -0.969004 & -0.398333 & 2.259853 \\
\hline 3 & 1 & -1.984098 & -0.416333 & 2.668651 \\
\hline 4 & 1 & -0.455483 & -1.290095 & 2.623706 \\
\hline 5 & 1 & -0.450939 & 0.474601 & 2.664142 \\
\hline 6 & 6 & -1.008551 & -0.414388 & 0.722695 \\
\hline 7 & 6 & -1.591353 & -1.714896 & 0.093119 \\
\hline 8 & 6 & -1.805787 & 0.639571 & 0.100687 \\
\hline 9 & 6 & -2.068034 & -1.297266 & -1.312167 \\
\hline 10 & 1 & -2.433696 & -2.055564 & 0.706410 \\
\hline 11 & 1 & -0.863489 & -2.527868 & 0.075670 \\
\hline 12 & 6 & -2.480218 & 0.186035 & -1.144016 \\
\hline 13 & 1 & -1.266201 & -1.393455 & -2.049138 \\
\hline 14 & 1 & -2.899771 & -1.909277 & -1.668236 \\
\hline 15 & 6 & -2.019674 & 1.977543 & 0.666196 \\
\hline 16 & 1 & -2.907461 & 1.878104 & 1.318501 \\
\hline 17 & 1 & -1.209468 & 2.307920 & 1.317239 \\
\hline 18 & 1 & -2.265627 & 2.730175 & -0.086790 \\
\hline 19 & 6 & -4.027863 & 0.381171 & -1.015363 \\
\hline 20 & 1 & -4.434913 & -0.210346 & -0.190489 \\
\hline 21 & 1 & -4.299956 & 1.429242 & -0.873160 \\
\hline 22 & 1 & -4.484255 & 0.031522 & -1.943824 \\
\hline
\end{tabular}




$\begin{array}{lllll}23 & 6 & 1.067848 & 1.255283 & 0.172804 \\ 24 & 6 & 1.494372 & -1.151449 & 0.734248 \\ 25 & 6 & 2.523059 & 1.304791 & -0.240481 \\ 26 & 6 & 2.804994 & -1.157245 & -0.070995 \\ 27 & 6 & 3.316555 & 0.232445 & -0.378984 \\ 28 & 1 & 0.409292 & -0.429768 & -0.977832 \\ 29 & 1 & 1.094264 & -2.167598 & 0.788655 \\ 30 & 1 & 1.705359 & -0.825813 & 1.758438 \\ 31 & 1 & 3.560301 & -1.708490 & 0.504316 \\ 32 & 1 & 2.681249 & -1.721462 & -1.007444 \\ 33 & 1 & 2.919649 & 2.297341 & -0.443547 \\ 34 & 1 & 0.504855 & 1.940449 & -0.475344 \\ 35 & 1 & 0.968388 & 1.651300 & 1.195471 \\ 36 & 6 & 4.745614 & 0.333517 & -0.841975 \\ 37 & 1 & 5.023074 & 1.362599 & -1.083359 \\ 38 & 1 & 5.434213 & -0.036417 & -0.072182 \\ 39 & 1 & 4.911421 & -0.283034 & -1.734548 \\ 40 & 1 & -2.161786 & 0.835124 & -1.974884 \\ ------------------------------------------------\end{array}$

14] TS B-to-cuprenyl-II

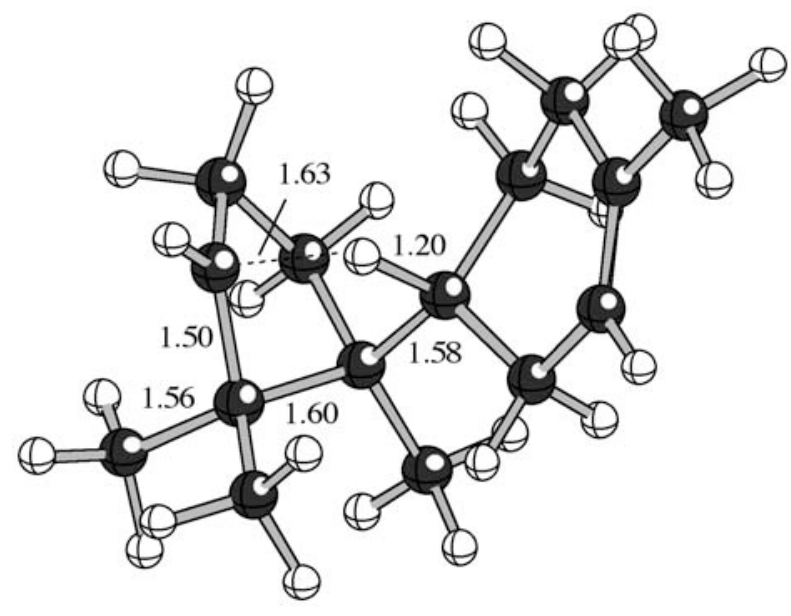

B3LYP/6-31+G[d,p]:-586.3726072 hartrees [-367948.811018 kcal/mol] Imaginary frequencies: $1[-303.73921 / \mathrm{cm}]$

Zero-point correction $=0.363557$ (Hartree/Particle $)$

mPW1PW91/6-31+G[d,p]//B3LYP/6-31+G[d,p]: -586.2473691 hartrees [-367870.22411025 kcal/mol]

Coordinates:

\begin{tabular}{lllll} 
Center & Atomic & \multicolumn{3}{c}{ Coordinates [Angstroms] } \\
number & number & X & $Y$ & Z \\
\hline 1 & 6 & -0.251328 & 0.069510 & 0.365562 \\
2 & 6 & 1.640446 & -0.480474 & 2.106278
\end{tabular}




\begin{tabular}{|c|c|c|c|}
\hline 3 & 1 & 2.713399 & $-0.380560 \quad 2.297449$ \\
\hline 4 & 1 & 1.119768 & $0.045561 \quad 2.913496$ \\
\hline 5 & 1 & 1.383383 & $-1.540848 \quad 2.179452$ \\
\hline 6 & 6 & 1.284735 & $0.111548 \quad 0.749892$ \\
\hline 7 & 6 & 1.736232 & $1.590344 \quad 0.574078$ \\
\hline 8 & 6 & 1.945516 & $-0.555961-0.542078$ \\
\hline 9 & 6 & 1.515159 & $1.822252-0.947458$ \\
\hline 10 & 1 & 2.782239 & $1.715833 \quad 0.856350$ \\
\hline 11 & 1 & 1.168150 & 2.2825201 .194806 \\
\hline 12 & 6 & 1.312272 & $0.444565-1.465302$ \\
\hline 13 & 1 & 0.701800 & $2.504082-1.215666$ \\
\hline 14 & 1 & 2.403176 & $2.219393-1.467984$ \\
\hline 15 & 6 & 1.610961 & $-2.016940-0.908790$ \\
\hline 16 & 1 & 2.276338 & $-2.350958-1.710180$ \\
\hline 17 & 1 & 1.775057 & $-2.682295-0.056906$ \\
\hline 18 & 1 & 0.590812 & $-2.154642-1.268343$ \\
\hline 19 & 6 & 3.500240 & $-0.434289-0.608098$ \\
\hline 20 & 1 & 3.921743 & $-1.160382 \quad 0.093708$ \\
\hline 21 & 1 & 3.861009 & $-0.699667-1.605780$ \\
\hline 22 & 1 & 3.891791 & $0.550415-0.356504$ \\
\hline 23 & 6 & -1.005316 & $-1.228093 \quad 0.689501$ \\
\hline 24 & 6 & -1.170823 & $1.243716 \quad 0.716594$ \\
\hline 25 & 6 & -2.428702 & $-1.257645 \quad 0.190734$ \\
\hline 26 & 6 & -2.495984 & $1.206713-0.069246$ \\
\hline 27 & 6 & -3.121609 & $-0.166898-0.166726$ \\
\hline 28 & 1 & -0.155213 & $0.119771-0.832990$ \\
\hline 29 & 1 & -0.702356 & $2.217677 \quad 0.570132$ \\
\hline 30 & 1 & -1.379527 & $1.166351 \quad 1.793552$ \\
\hline 31 & 1 & -3.201389 & $1.899097 \quad 0.406410$ \\
\hline 32 & 1 & -2.344449 & $1.607879-1.083924$ \\
\hline 33 & 1 & -2.894232 & $-2.238744 \quad 0.132917$ \\
\hline 34 & 1 & -0.475821 & $-2.122872 \quad 0.370221$ \\
\hline 35 & 1 & -0.994467 & -1.2769591 .791758 \\
\hline 36 & 6 & -4.531622 & $-0.226531-0.688601$ \\
\hline 37 & 1 & -4.889557 & $-1.255522-0.772722$ \\
\hline 38 & 1 & -5.215915 & $0.321517-0.029056$ \\
\hline 39 & 1 & -4.605387 & $0.243164-1.677771$ \\
\hline 40 & 1 & 1.028574 & $0.239291-2.500474$ \\
\hline
\end{tabular}

IRC: TS[B-to-cuprenyl-II]-> B 


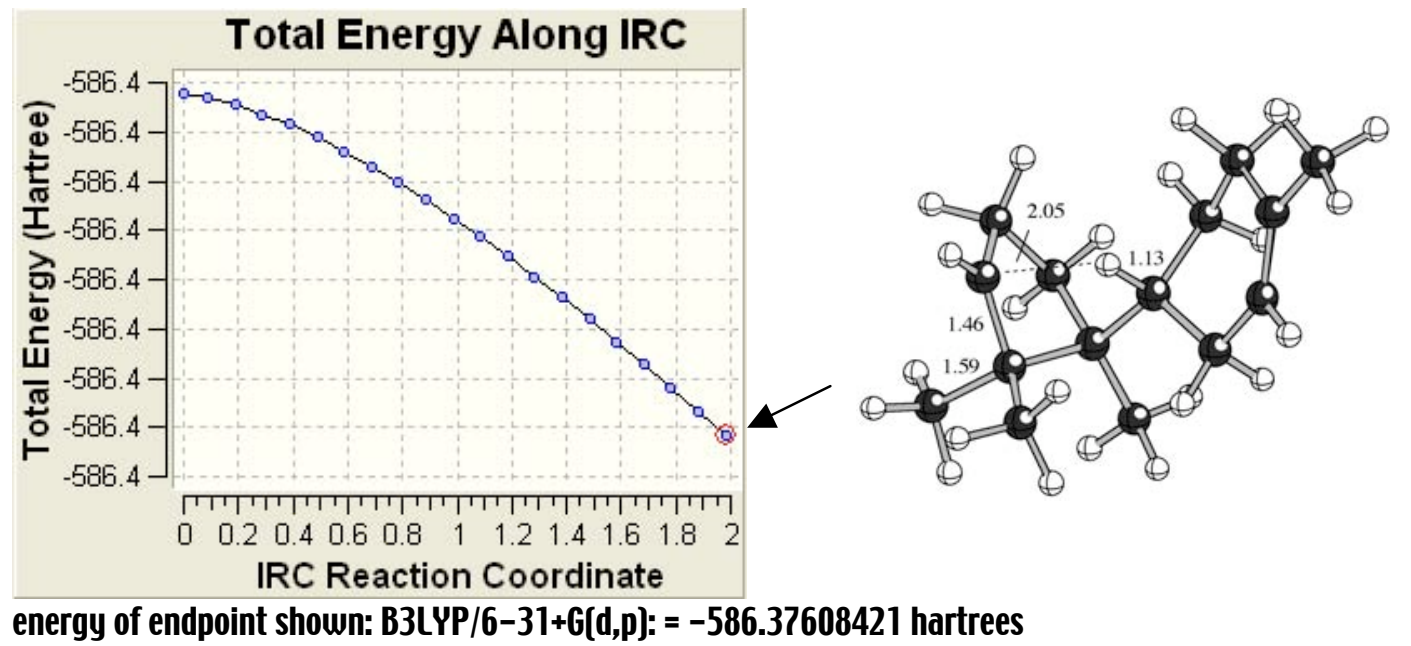

IRC: TS[B-to-cuprenyl-II]-> cuprenyl-II

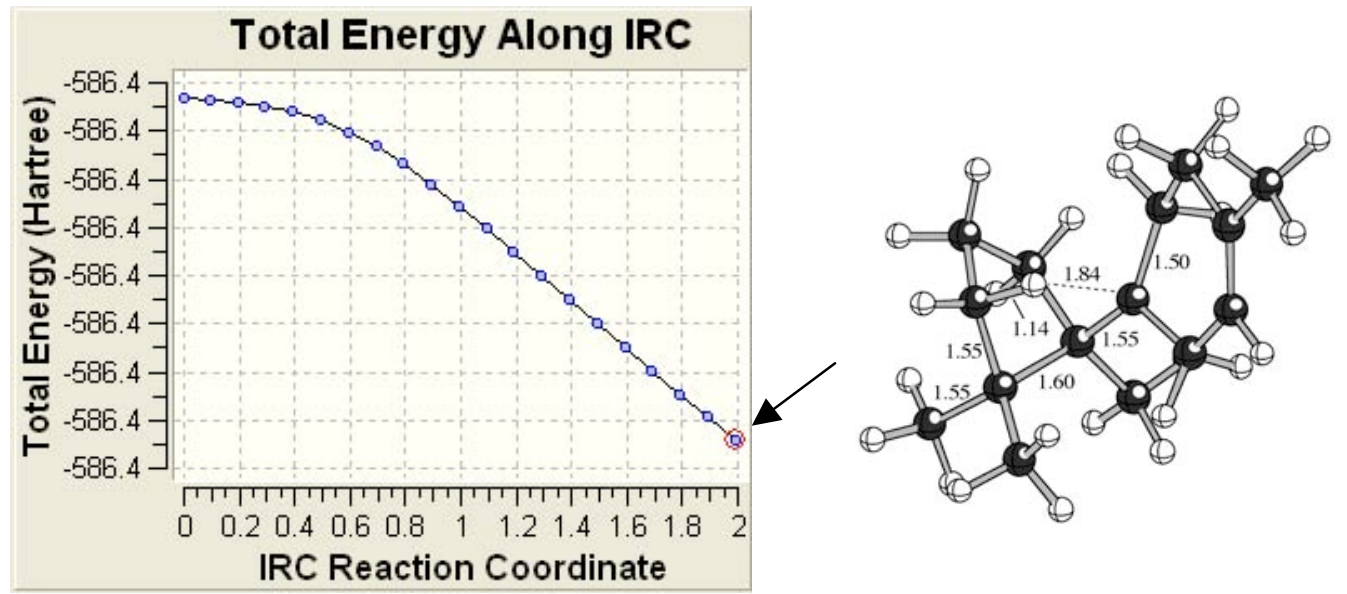

15) Cuprenyl-II

B3LYP/6-31+G[d,p]: -586.4181428 hartrees [-367977.384607 kcal/mol] Imaginary frequencies: none

Zero-point correction = 0.366199 (Hartree/Particle)

mPU1PW91/6-31+G[d,p]//B3LYP/6-31+G[d,p]: -586.289635 hartrees [-367896.7459625 kcal/mol]

Coordinates:

\begin{tabular}{lllll} 
Center & \multicolumn{1}{c}{ Atomic } & \multicolumn{3}{c}{ Coordinates [Angstroms] } \\
number & number & $X$ & $Y$ & $Z$ \\
\hline 1 & 6 & 0.280553 & -0.272378 & -0.647829 \\
2 & 6 & -1.596000 & -1.851705 & -1.134618 \\
3 & 1 & -2.681273 & -1.912128 & -1.206448 \\
4 & 1 & -1.195362 & -2.066356 & -2.131013
\end{tabular}




$\begin{array}{ccccc}5 & 1 & -1.266398 & -2.644125 & -0.458368 \\ 6 & 6 & -1.175050 & -0.438270 & -0.681039 \\ 7 & 6 & -1.913941 & 0.687920 & -1.479739 \\ 8 & 6 & -1.683243 & -0.101731 & 0.907161 \\ 9 & 6 & -2.293128 & 1.841037 & -0.509930 \\ 10 & 1 & -2.809583 & 0.241990 & -1.918464 \\ 11 & 1 & -1.313187 & 1.033719 & -2.325993 \\ 12 & 6 & -1.777202 & 1.435354 & 0.884101 \\ 13 & 1 & -1.874096 & 2.800020 & -0.828864 \\ 14 & 1 & -3.377984 & 1.975084 & -0.497793 \\ 15 & 6 & -0.760587 & -0.630245 & 2.011180 \\ 16 & 1 & -1.203154 & -0.395971 & 2.984636 \\ 17 & 1 & -0.647311 & -1.718878 & 1.970993 \\ 18 & 1 & 0.236864 & -0.178187 & 1.992845 \\ 19 & 6 & -3.079975 & -0.722662 & 1.079437 \\ 20 & 1 & -3.053291 & -1.813946 & 1.108757 \\ 21 & 1 & -3.473062 & -0.380403 & 2.043306 \\ 22 & 1 & -3.788507 & -0.409952 & 0.308544 \\ 23 & 6 & 1.187418 & -1.432364 & -0.503407 \\ 24 & 6 & 0.957222 & 1.026282 & -0.848775 \\ 25 & 6 & 2.627486 & -1.150575 & -0.165377 \\ 26 & 6 & 2.197815 & 1.272638 & 0.038434 \\ 27 & 6 & 3.107033 & 0.068004 & 0.119534 \\ 28 & 1 & -0.797119 & 1.878468 & 1.091097 \\ 29 & 1 & 0.276472 & 1.875010 & -0.857139 \\ 30 & 1 & 1.324222 & 0.917103 & -1.891886 \\ 31 & 1 & 2.738156 & 2.127805 & -0.380864 \\ 32 & 1 & 1.888510 & 1.583864 & 1.046186 \\ 33 & 1 & 3.273028 & -2.022988 & -0.114398 \\ 34 & 1 & 0.745912 & -2.168924 & 0.181330 \\ 35 & 1 & 1.109361 & -1.951924 & -1.481524 \\ 36 & 6 & 4.527087 & 0.314875 & 0.546712 \\ 37 & 1 & 5.094080 & -0.615465 & 0.624483 \\ 38 & 1 & 5.040274 & 0.974023 & -0.163917 \\ 39 & 1 & 4.557685 & 0.816955 & 1.521925 \\ 40 & 1 & -2.437168 & 1.778126 & 1.687495 \\ ------------------------------------------------------- \\ -\end{array}$

